دراسة تحليلية لوضع الأمن الغذائي للسلع الغذائية الحيو انية في مصر

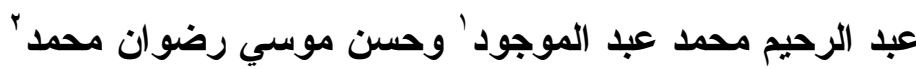

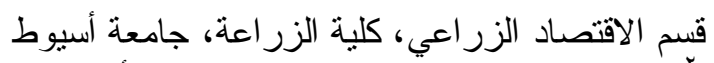

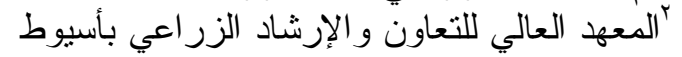

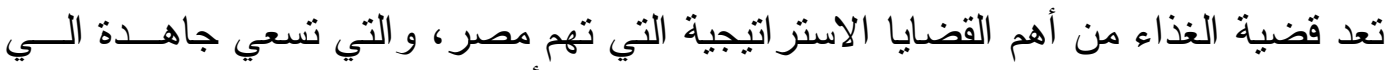

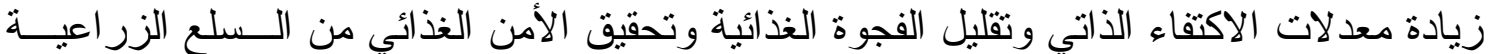

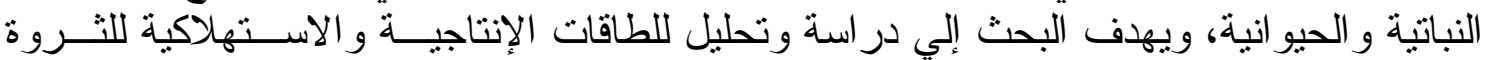

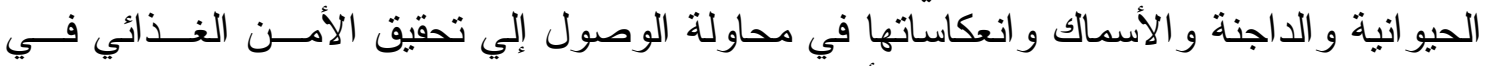

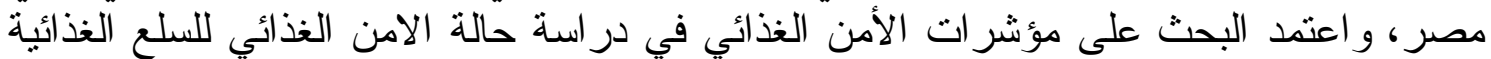

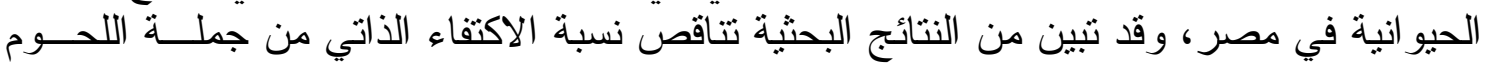

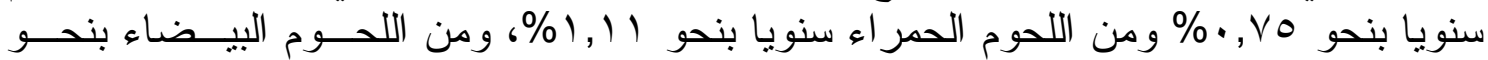

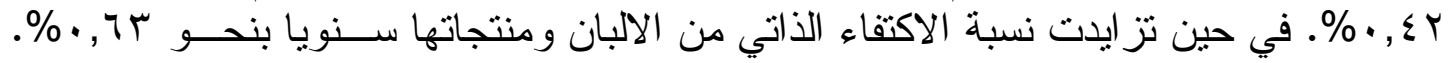

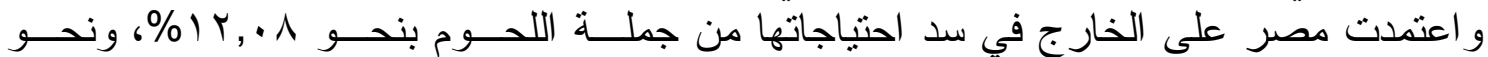

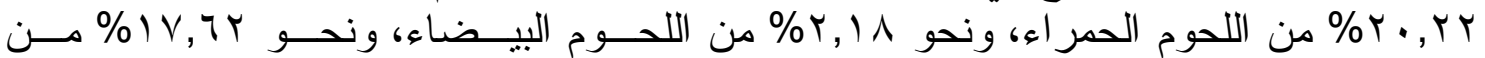

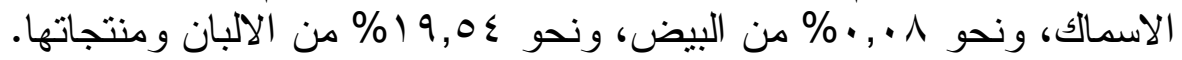

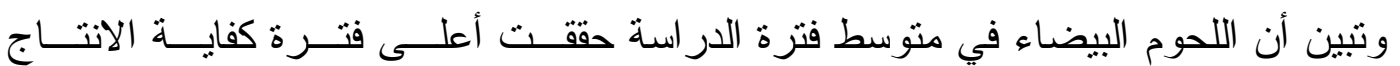

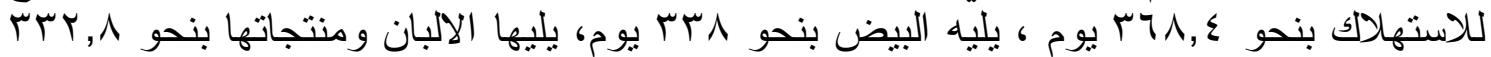

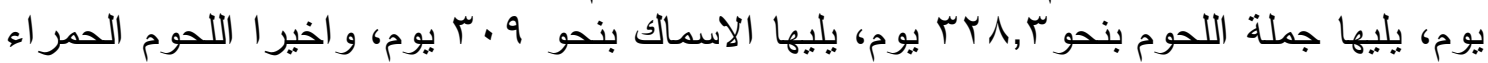

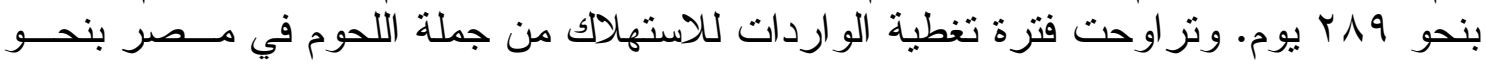

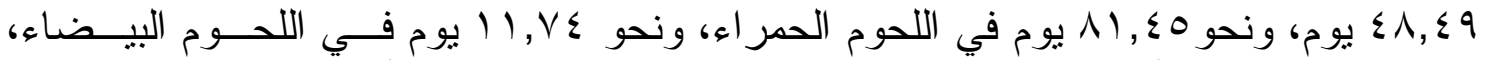

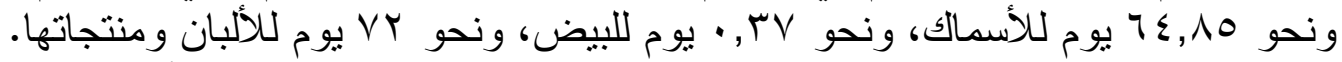

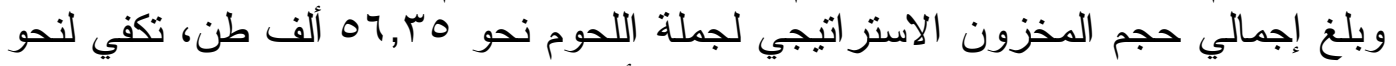

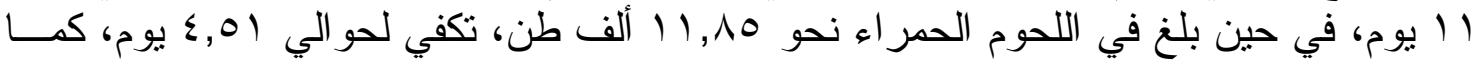

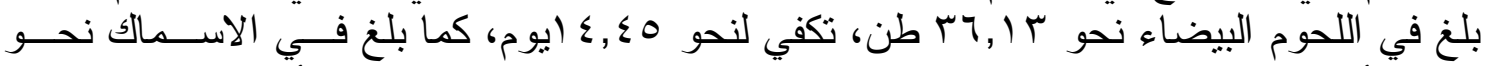


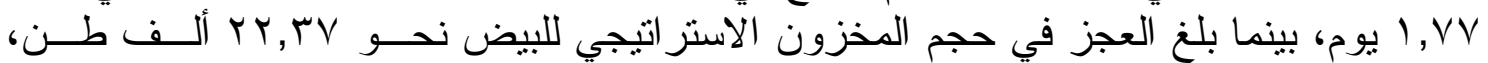

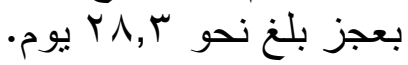

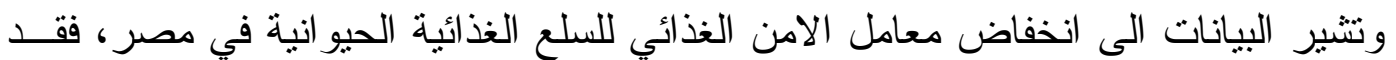

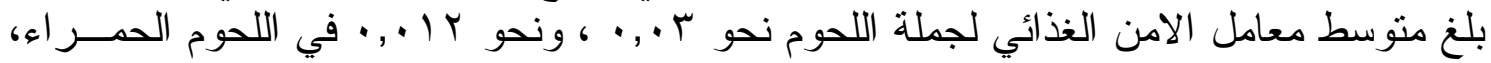

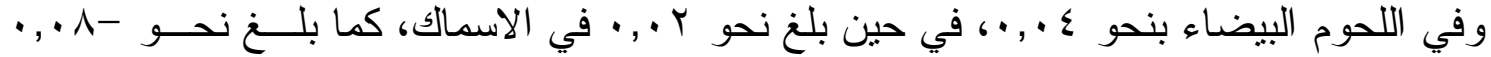

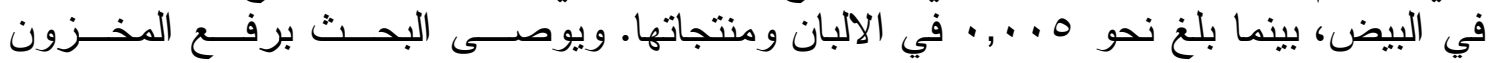

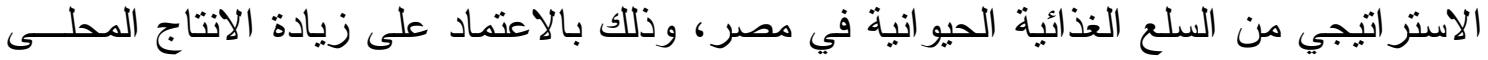

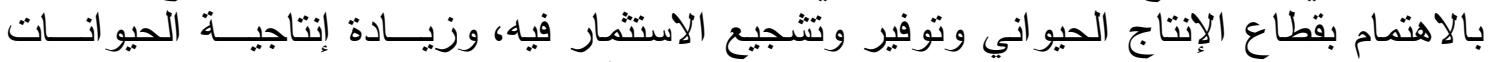

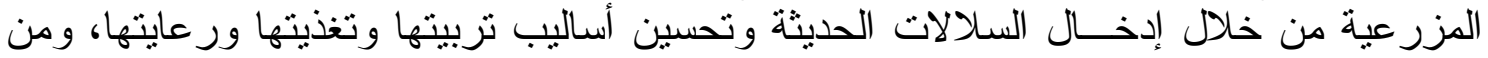

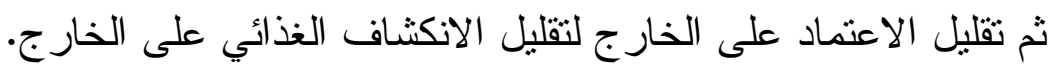

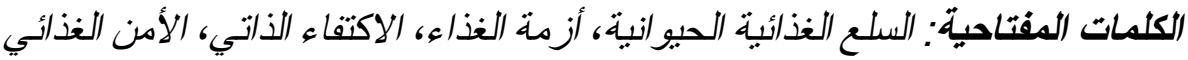




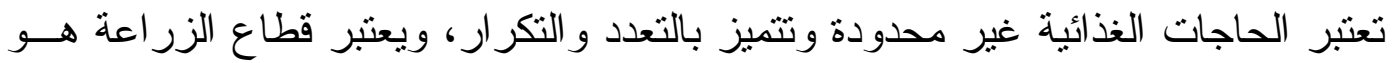

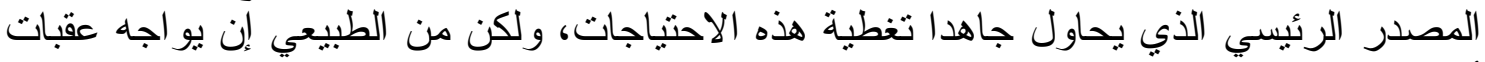

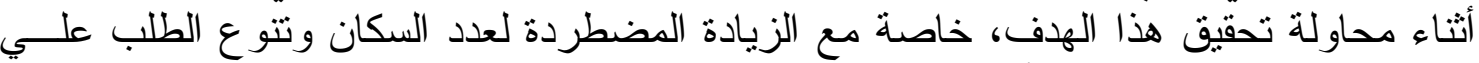

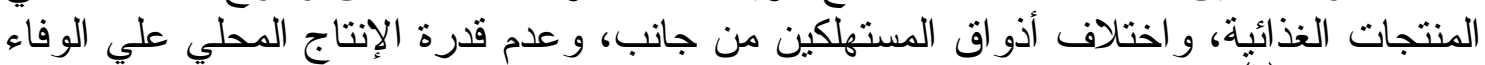

بهذه الحاجات(7).

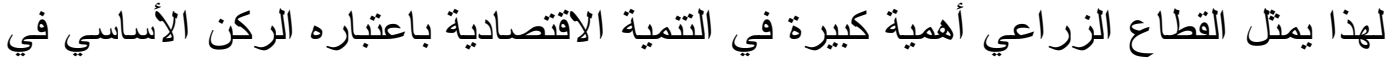

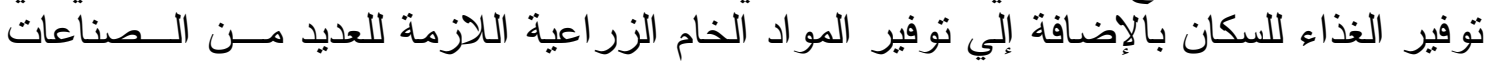

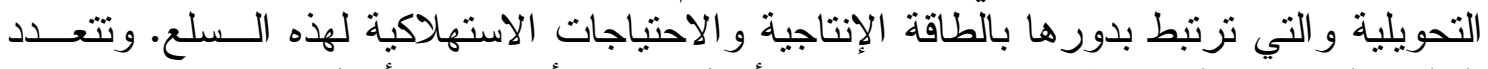

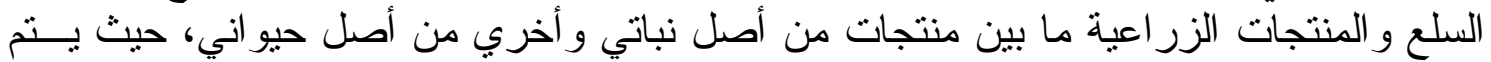

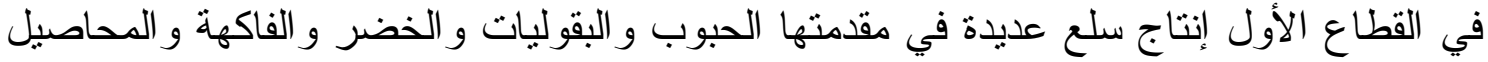

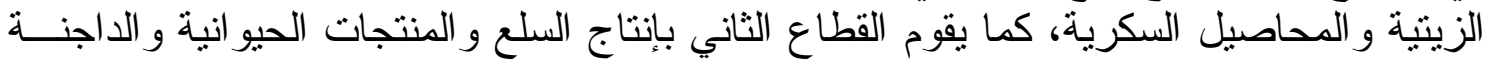

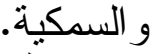
ظّهر مفهوم الأمن الغذائي في إعقاب أزمة الغذاء العالمية في النصف الأول من السبعينات

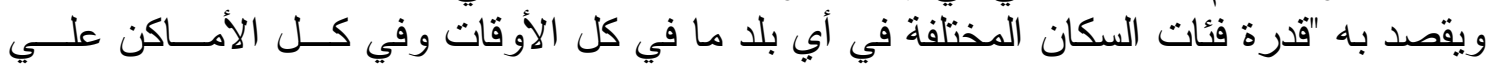

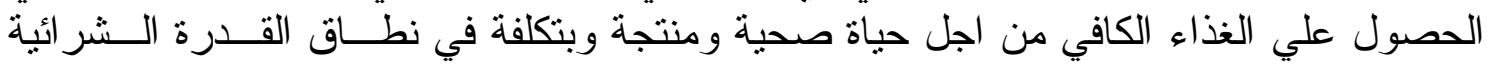

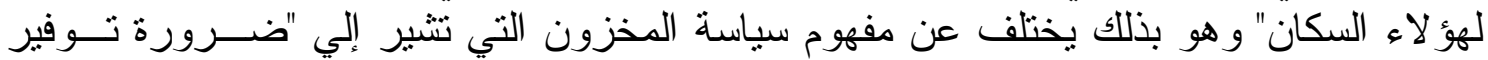

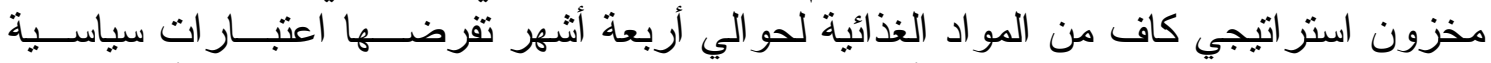

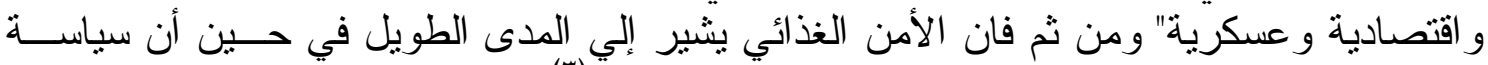

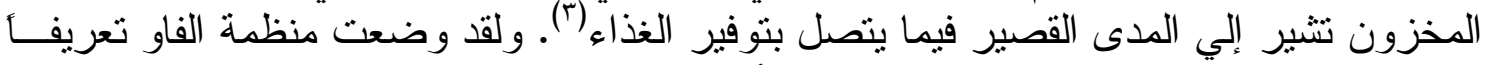

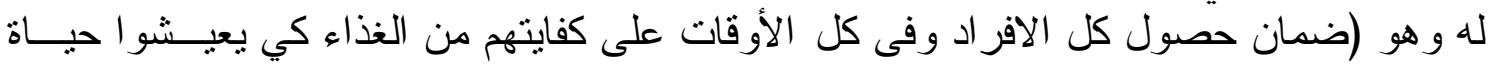

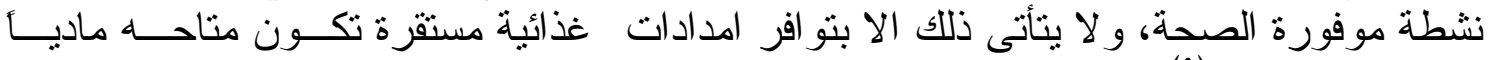

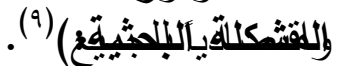

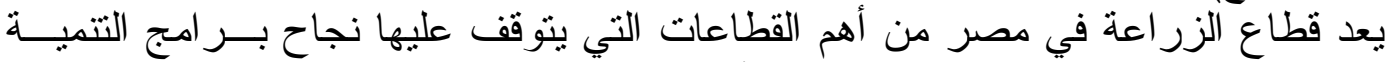

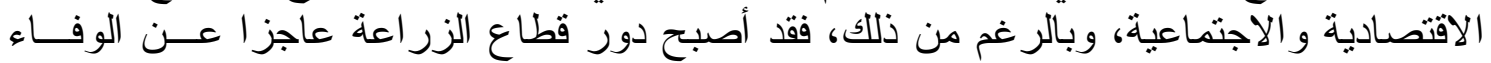

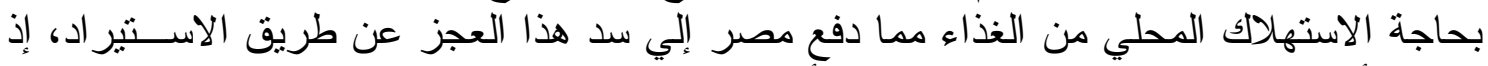

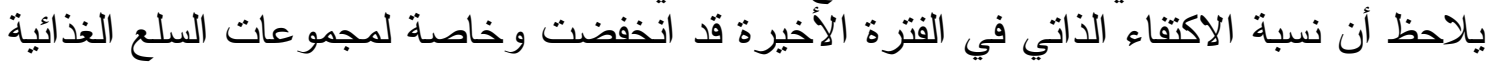

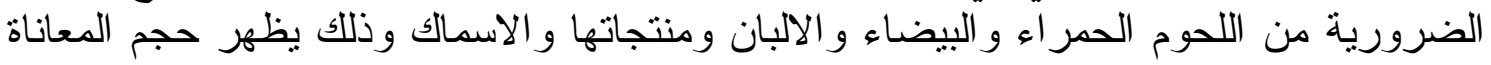

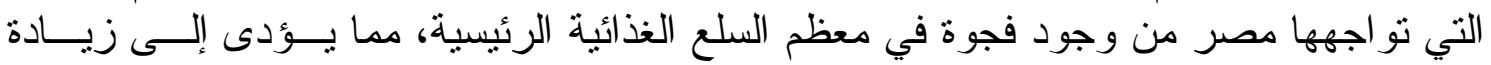
اعتمادها على الاستير اد من الخارج في سي سد معظم احتياجاتها الغذائية. الأهداف البحثية: يهدف البحث إلي الوقوف علي الوضع الرافي الهن و المستقبلي للطاقات الإنتاجية و الاستهلاكية

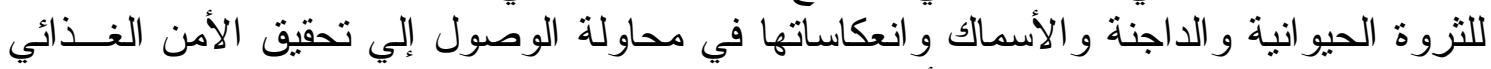

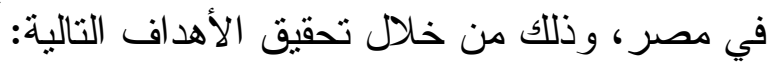

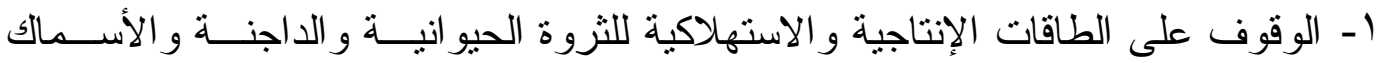
ونوقعاتها المستقبلية.

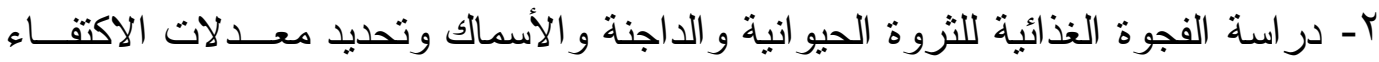
الذاتي منها وتوقعاتها المستقبلية.

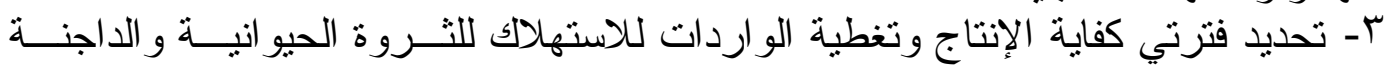

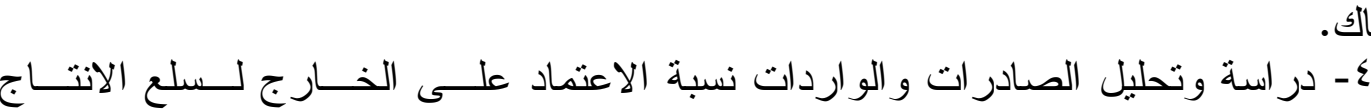
و الأسمالك. الحيو اني. 
0ـ إلقاء الضوء علي الأسباب المتوقعة ور اء الفجوة الغذائية ومقترحسـات زيــادة معـلـل الاكتفاء الذاتي للثروة الحيؤاءنية و الداجنة و الأسماك.

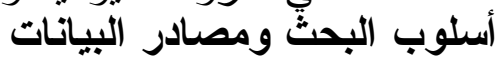

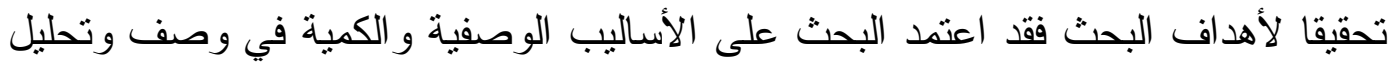

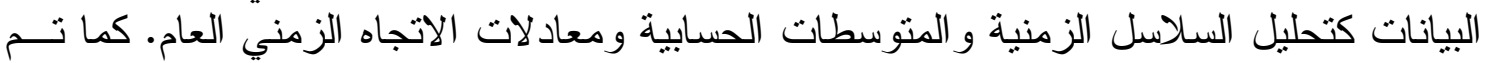

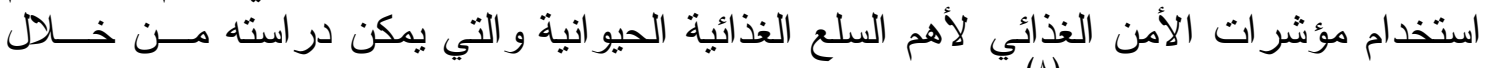

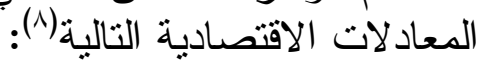

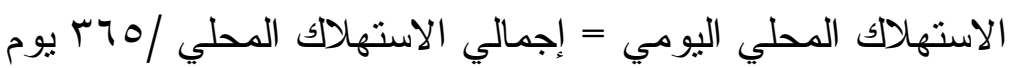

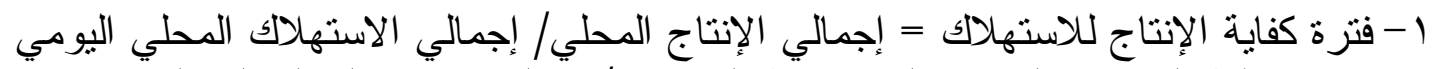

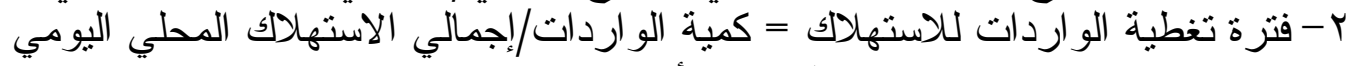

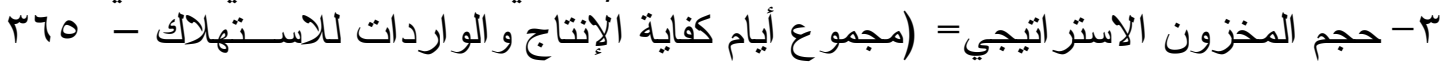

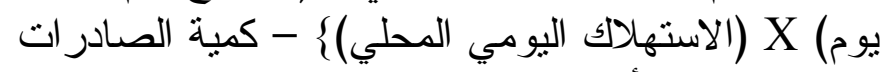

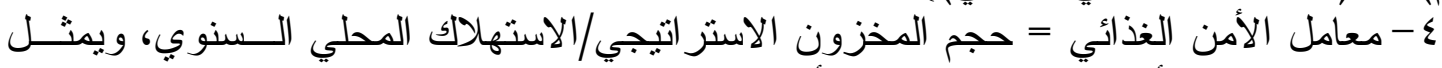

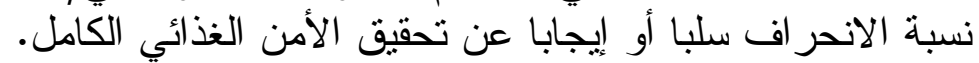

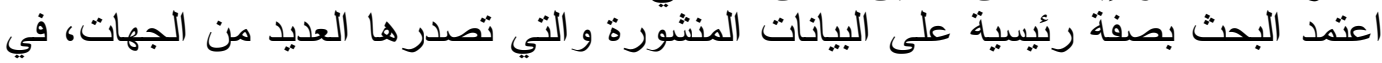

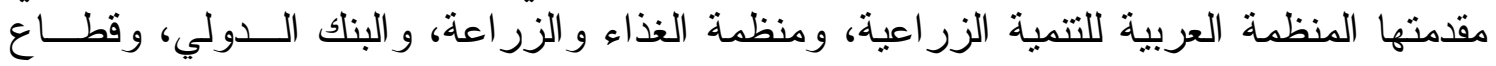

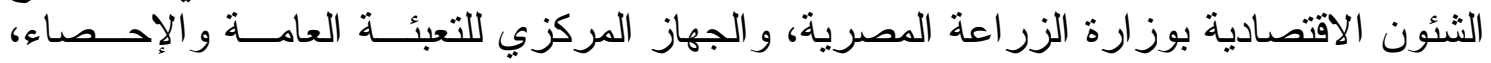

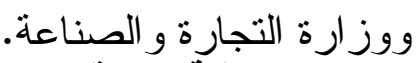
النتائج البحثية ومناقشتها

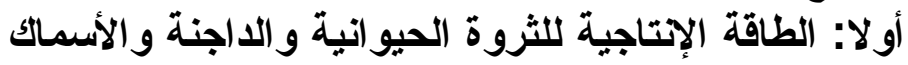

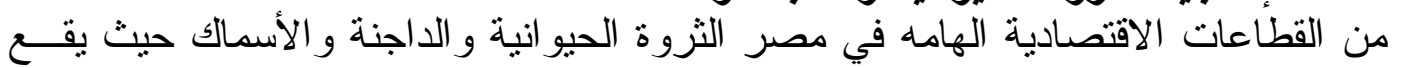

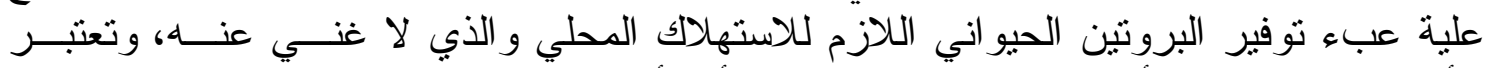

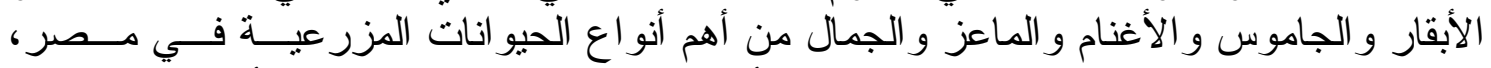

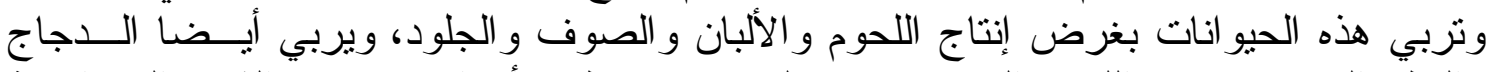

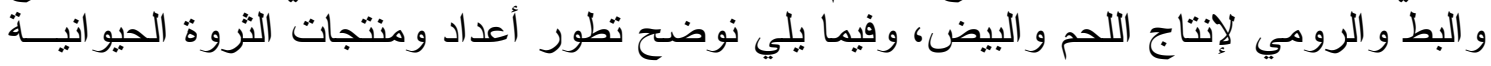

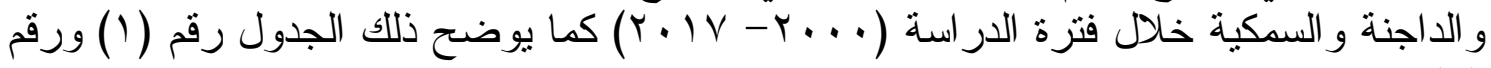

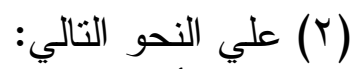
1 - أطوز أعداد الحيوانات:

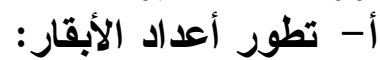

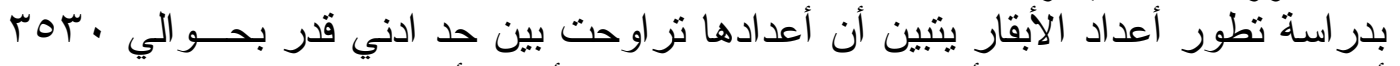

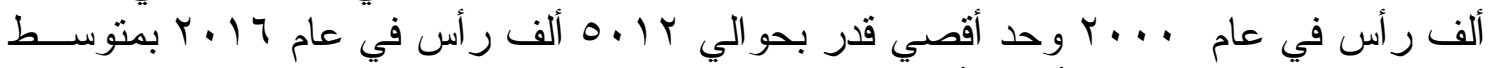

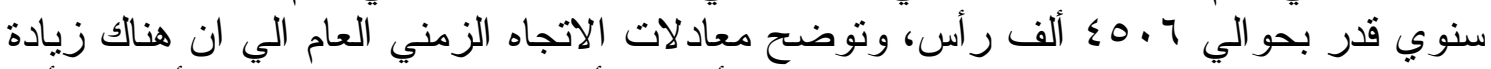

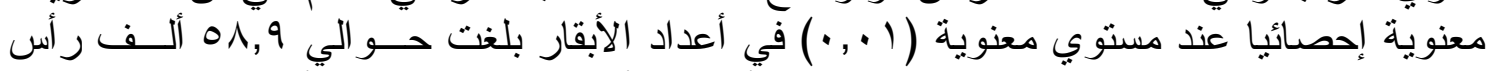

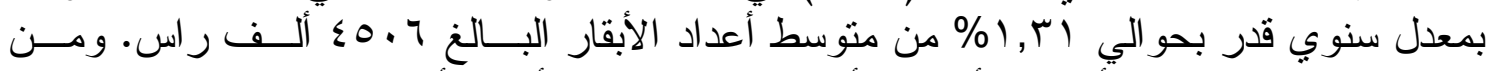

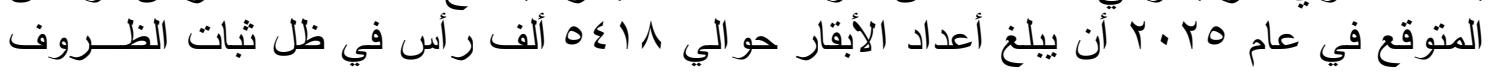

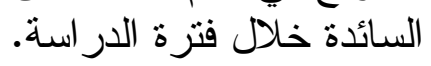

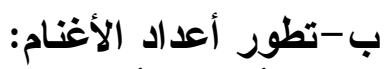

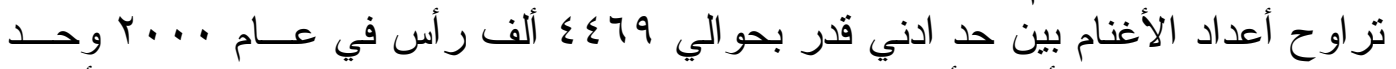

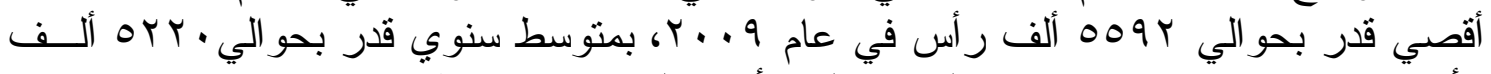

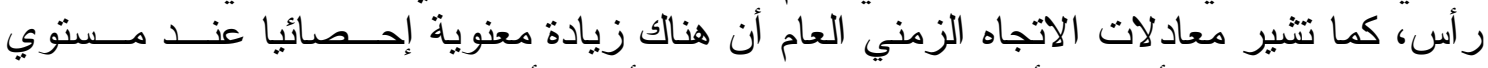

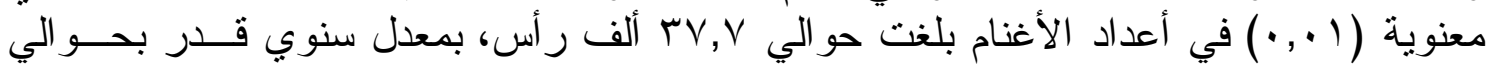




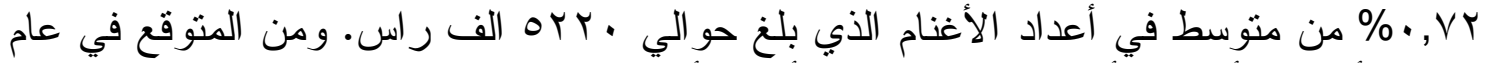

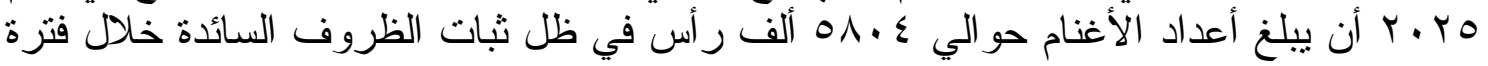
الدار اسة.

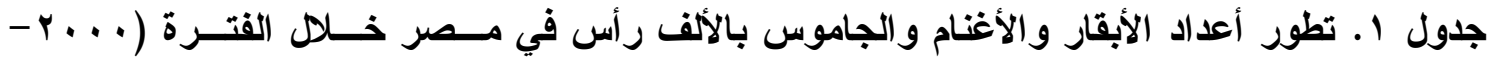

\begin{tabular}{|c|c|c|c|c|c|c|c|c|c|c|}
\hline الاسمـاك & البيض & الألبان & الليضوم الليضاء & الحمر اءء & الماعز & الجمال & الجاموس & الأغتام & الأبَقار & السنو ات \\
\hline$V Y \varepsilon$ & $T \leq \varepsilon$ & พัィイ & $7 \leqslant 1$ & $7 . \varepsilon$ & $r \leqslant Y O$ & $1 \leqslant 1$ & Tr人E & $\varepsilon \leqslant 79$ & ror. & $r \ldots$ \\
\hline VTV & roq & rAM & 117 & 711 & $r \leqslant q V$ & $1 \pi \varepsilon$ & rorr & $\varepsilon T V 1$ & rᄉ.. & $r \ldots 1$ \\
\hline VTV & ror & $\varepsilon \cdot \wedge \varepsilon$ & $1 \cdot 1$. & $7 . V$ & TONT & ITV & rVIV & 01.0 & $\varepsilon \cdot \lambda l$ & $r \ldots r$ \\
\hline AVI & TOY & $01 \leqslant \lambda$ & Nrq & VAr & rNI & $1 \pi 4$ & TVVV & $\varepsilon q \uparrow q$ & EYYO & $r \ldots r$ \\
\hline ヘTร & TI & $\varepsilon r q r$ & 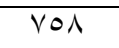 & TYV & rAVq & 119 & $r \wedge \leqslant 0$ & $0 . \leqslant \mu$ & $\varepsilon r 7 q$ & $r \ldots \varepsilon$ \\
\hline 170 & YI. & 0799 & NTI & 974 & $r 910$ & $1 \pi 1$ & 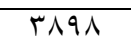 & $0.9 \mathrm{~V}$ & §६। & $r \ldots o$ \\
\hline $9 \times 1$ & $19 \varepsilon$ & 0709 & 717 & VVY & rq7. & $1 \leqslant 1$ & rquV & $0 \% \wedge 0$ & $\{7)$. & $r \ldots r$ \\
\hline $1 \cdots v$ & YYI & ovV. & 77. & $V \cdot 7$ & 1917 & $10 \mathrm{~V}$ & rqVV & $00 Y 0$ & $\leqslant 0 \leqslant \Lambda$ & $r \ldots V$ \\
\hline $1.7 V$ & Mo & 0995 & 009 & $\vee \wedge \varepsilon$ & EYMV & $19 r$ & \&10r & $0 \leqslant 91$ & $\varepsilon \wedge 0 \wedge$ & $r \ldots \lambda$ \\
\hline 1.94 & TIY & OTYE & $\Lambda \cdot \Lambda$ & 971 & $\sum \backslash r q$ & $1 T V$ & rᄉrq & $009 r$ & ¿รYO & $r \ldots q$ \\
\hline $1 \pi \cdot 0$ & TVO & OVVE & Vor & דז' & हाV० & IrV & rᄉIA & OOY & $\sum V Y q$ & $r+1$. \\
\hline ITYY & TAN & $O \Lambda \cdot r$ & 1.9 & 110 & $\sum Y O \Lambda$ & $1 \varepsilon$. & rqAr & 0470 & $\Sigma \vee \wedge$. & $r .11$ \\
\hline$|r V|$ & rq1 & $0 \wedge \leqslant 9$ & $1 . r V$ & NIT & $\sum \Gamma \cdot \tau$ & $1 \leqslant Y$ & r910 & $\Sigma 99 \wedge$ & $\leq 9 \leq 7$ & $r \cdot I r$ \\
\hline ITVE & T97 & $000 \leqslant$ & $11 \wedge V$ & 297 & ह10r & 104 & $r 910$ & $\{919$ & $\leqslant V \leqslant 0$ & $r+1 r$ \\
\hline $1 \leqslant \lambda r$ & r97 & 07.1 & $1 Y \cdot T$ & 199 & $\left.\sum 1 \wedge\right\rceil$ & 101 & $r q \leq q$ & 00.4 & $\sum V 7 Y$ & $Y .1 \leq$ \\
\hline 1019 & rqT & $01 Y T$ & $I T \cdot V$ & $\Lambda) \varepsilon$ & $\varepsilon \cdot \leqslant 7$ & $10 Y$ & $r V \cdot r$ & $0 \leq 7 \pi$ & $\varepsilon \wedge \wedge \mu$ & $r+10$ \\
\hline $17 \varepsilon$. & $r \cdot \tau$ & 0.19 & 1419 & $\Lambda \cdot V$ & ¿YT. & $10 \mathrm{~V}$ & $r \leqslant r V$ & 0007 & $0.1 \mathrm{r}$ & $r .17$ \\
\hline IAYT & rq1 & OY07 & $107 Y$ & ヘTY & rqv & 170 & $r \leqslant r r$ & $0 \% .0$ & $\varepsilon r \wedge V$ & $r . I V$ \\
\hline 117. & YTV & OYIA & QY & V\%. & $\mathrm{q \wedge \varepsilon , \varepsilon}$ & $1 \leqslant 7, \varepsilon$ & rVqr,\& & OrY. & $\leqslant 0.7$ & المتوسط \\
\hline
\end{tabular}

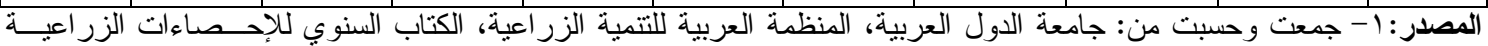
العربية، الخرطوم، أعداد مختلفة.

ץ- الجهاز المركزي للتعبئة العامة و الإحصاء، إحصاءات الثروة الحيو انية، بيانات منشوره، أعداد متفرقة.

\section{ج-تطور أعداد الجاموس:}

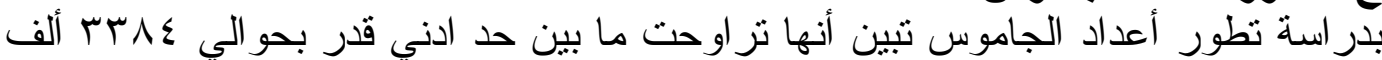

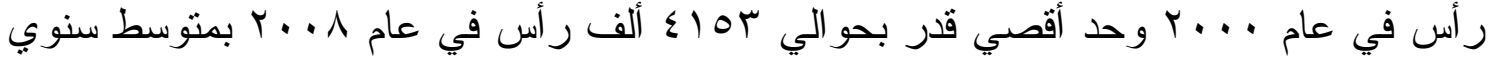

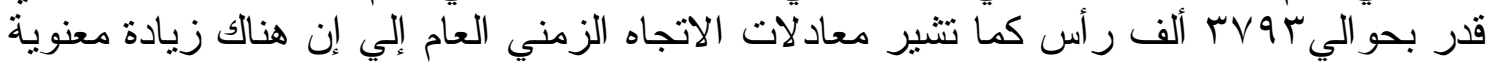

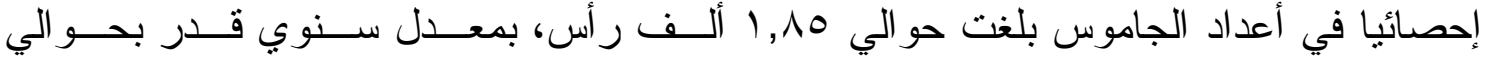

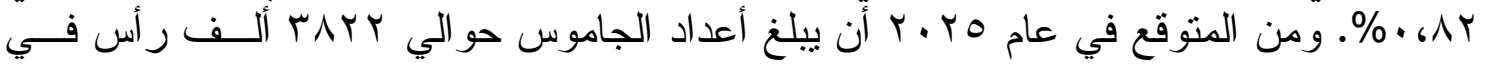
ظل ثبات الظروف السائدة خلاف فترة الدر اسة.

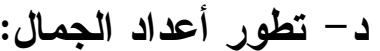

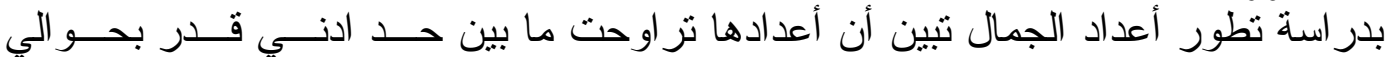

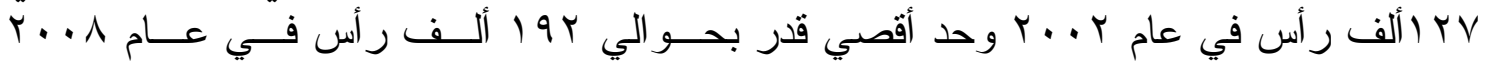

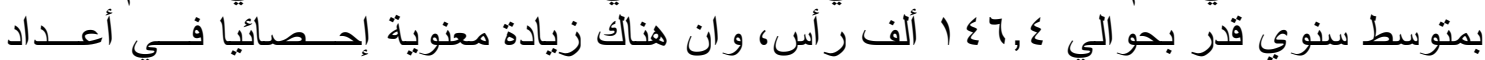

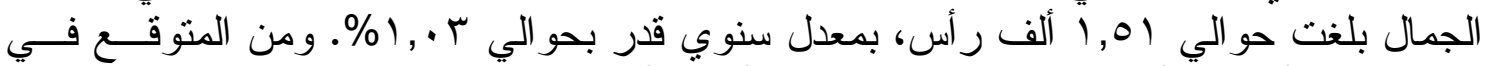

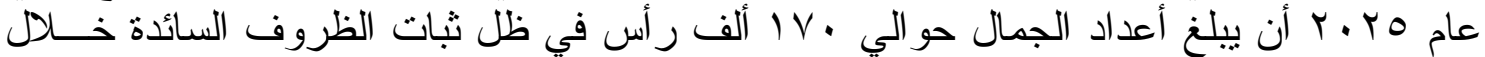
فتزة الدر اسة. 
Assiut J. Agric. Sci., 51 (2) 2020 (195-212)

ISSN: 1110-0486

Website:www.aun.edu.eg/faculty_agriculture/journals_issues_form.php E-mail: ajas@aun.edu.eg جدول r. معادلات الاتجاه الزمنـي العام لتطور أعداد الأبقار والأغنام والجــاموس بــالألف رأس فــي

\begin{tabular}{|c|c|c|c|c|c|c|}
\hline & & & & & 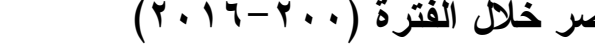 & \\
\hline 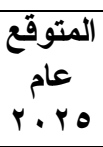 & ف & נر & السنوي \% معل التغير & المتوسط & النموذج & التابع \\
\hline $0 \leqslant 11$ & $* * Y_{0,70}$ & $\cdot, 7)$ & $1, \mu_{1}$ & $\{0.7$ & $\begin{array}{c}\omega 0 \Lambda, q+r q \leq 0, q={ }_{\Delta}^{\wedge} ص \\
* *(0, \cdot 7)\end{array}$ & الأبقار \\
\hline $0 \Lambda \cdot \varepsilon$ & $* * 9, \leqslant 7$ & $\cdot, T V Y$ & $\cdot, V Y$ & OrY. & 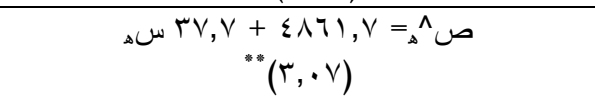 & الأغنام \\
\hline TAYT & ***r & $\cdot, \wedge r_{0}$ & $\cdot, \cdot 0$ & rVqT, \& & 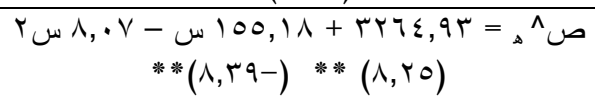 & الجاموس \\
\hline iv. & $* 0,0$ & , ro & $1, \cdot r$ & $1 \leq 7, \varepsilon$ & 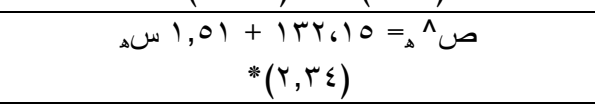 & الجمال \\
\hline$\{097$ & ***ץ, 0 & . & $1, \cdots$ & $r q \wedge \varepsilon, \varepsilon$ & 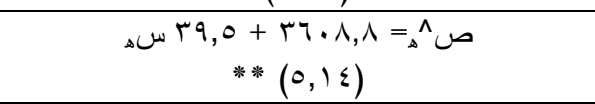 & الماعز \\
\hline $1 \ldots$ & $* * \backslash \wedge, \wedge q$ & $\cdot, 0 \leqslant 1$ & 1,97 & VI. & 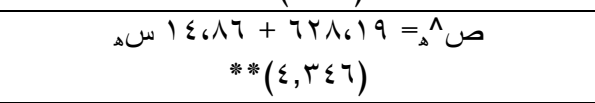 & 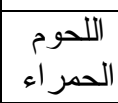 \\
\hline $1 \leqslant 79$ & $* * 10, \cdot V$ & $\cdot, \leqslant \wedge 0$ & $\ulcorner, \wedge \varepsilon$ & $9 T_{1}$ & 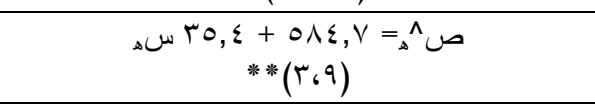 & البيضاء - اللحوم \\
\hline$T \leqslant r V$ & $* \vee, \wedge$ & - rrs & 1,01 & oril & 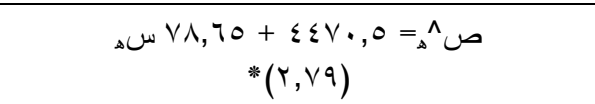 & الألبان \\
\hline- & $\cdot, \leqslant \Gamma$ & $\cdot, \cdot r$ & - & TTV & 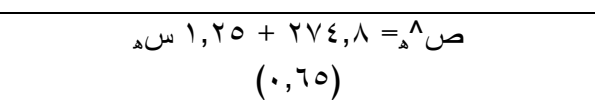 & البيض \\
\hline rI.V & $* * \varepsilon .0,7$ & $\cdot, 97$ & $0, r V$ & 117. & 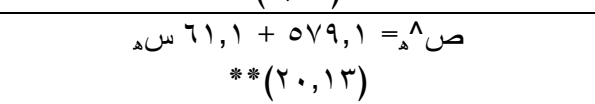 & الاسماك \\
\hline
\end{tabular}

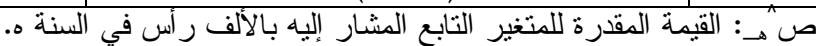

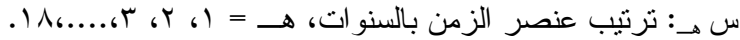

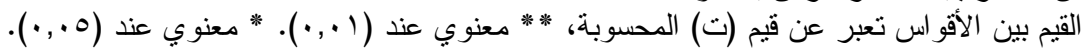
المصدر: حسبت من بيانات الجدول رقم (1).

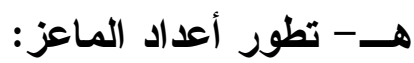

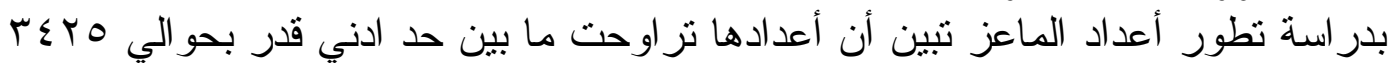

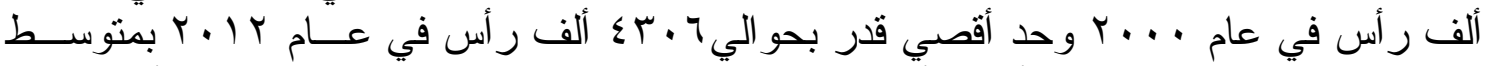

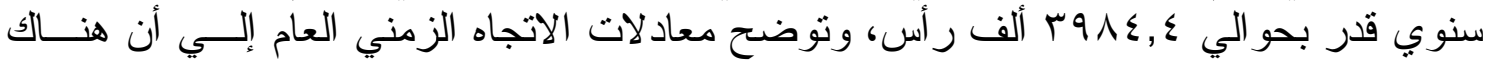

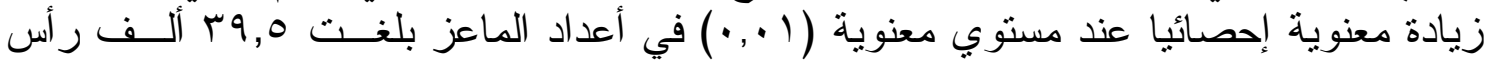

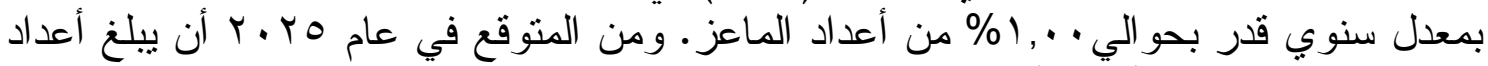

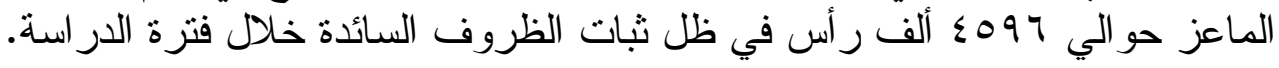

\section{r - المنتجات الحيو انية و الاجنة و السمكية: أ-تطور إنتاج اللحوم الحمر اع: ألهو:}

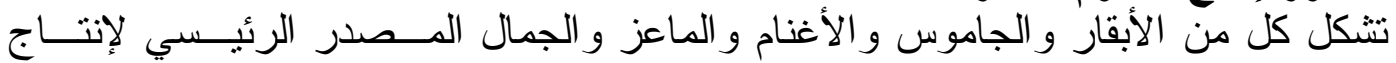

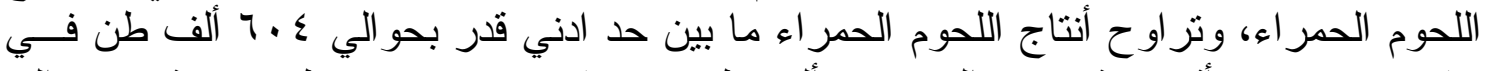

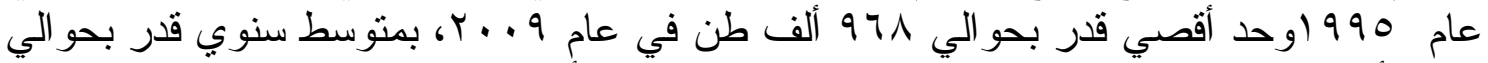
•

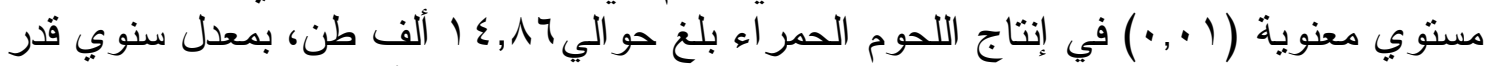

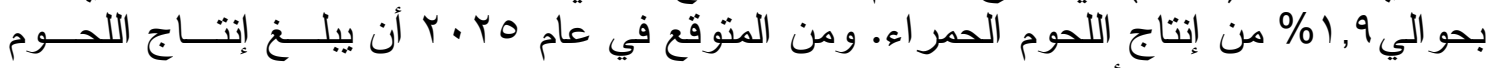

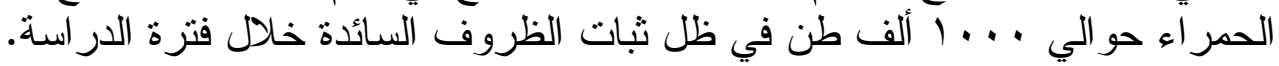


ب- تطور إنتاج اللحوم البيضاء:

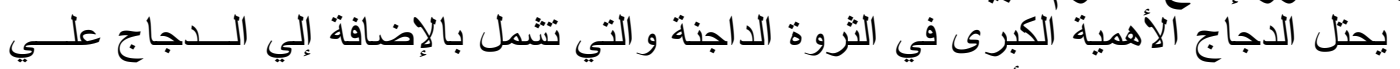

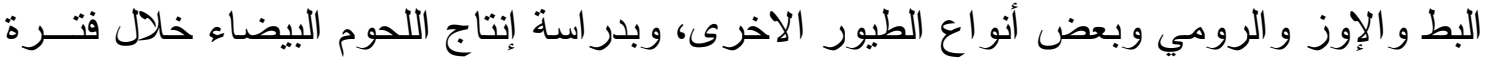

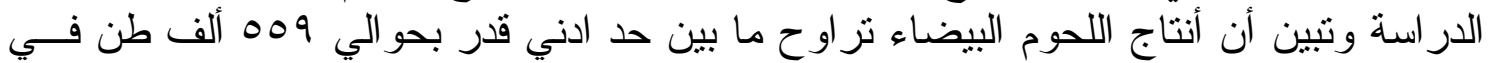

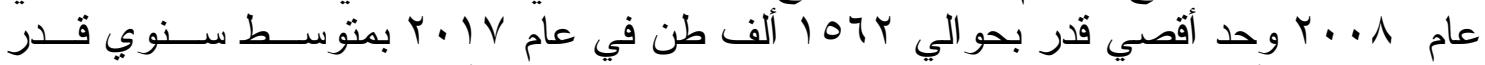

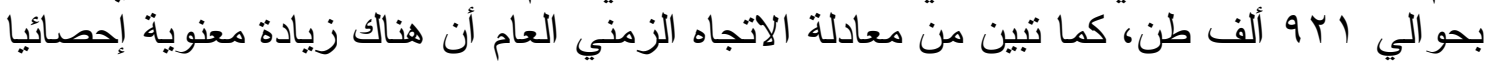

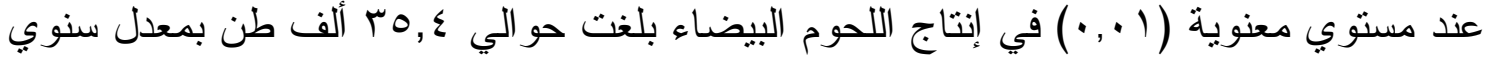

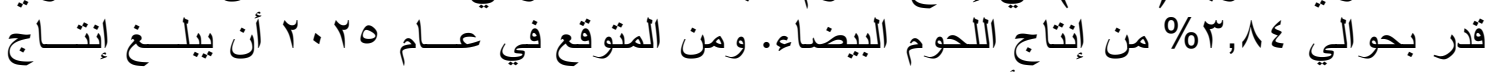
اللحوم البيضاء حو الي 979 ـ ألف طن في ظل ثبات الظروف السائدة خلال فترة الدر اسة.

\section{ج- تطور إنتاج الألبان:}

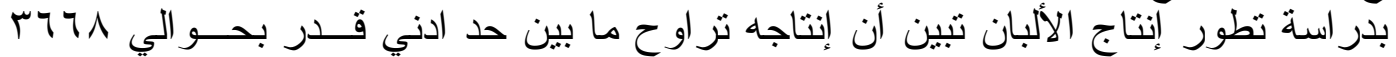

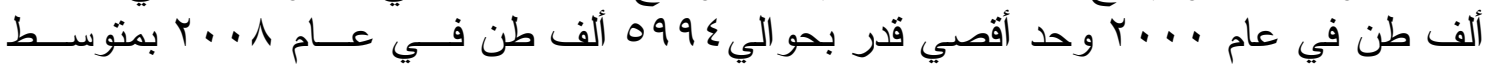

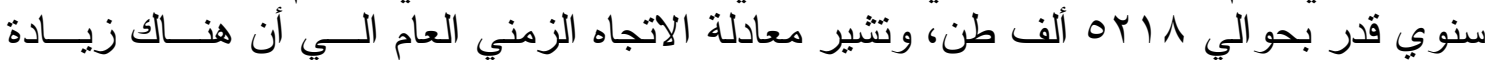

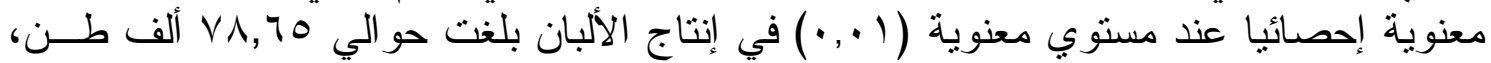

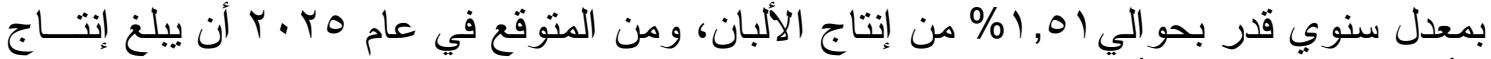

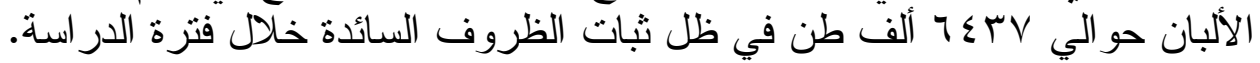

\section{د - تطور إنتاج البيض: تون}

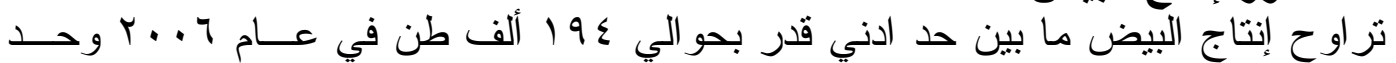

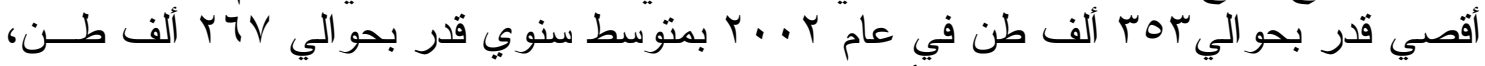

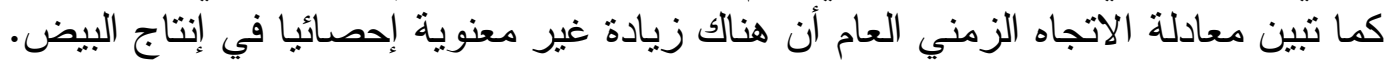

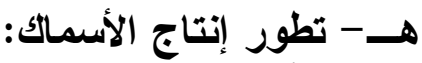

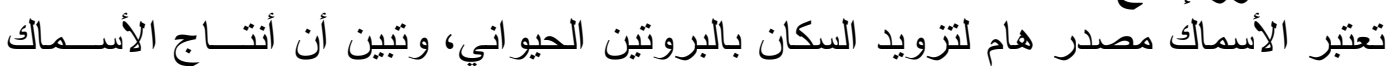

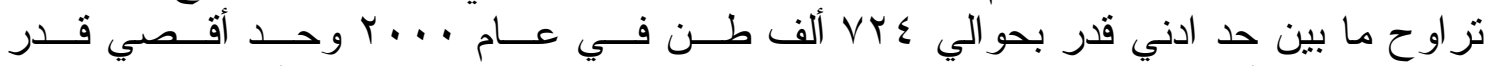

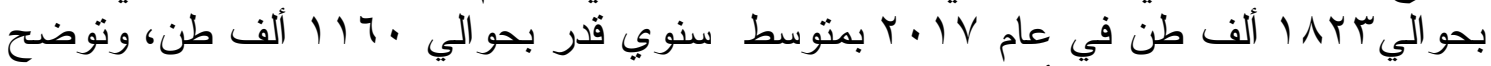

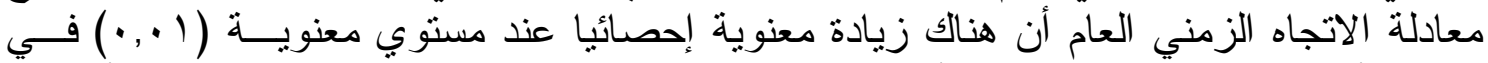

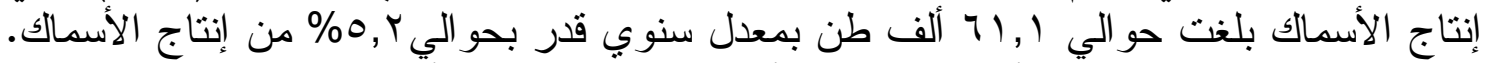

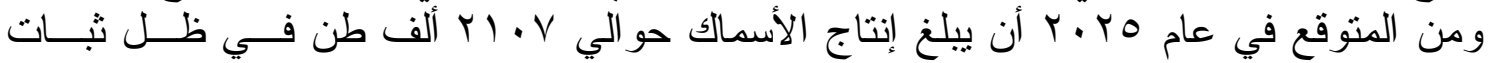

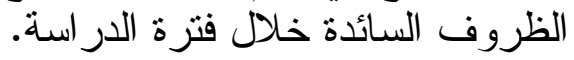

ثاتيا: الطاقة الاستهلاكية ونسبة الاكتفاء الذاتي للثروة الحيواتية والاجنة و الأسماك

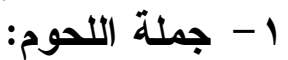

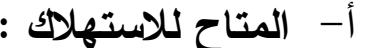

تز اوح استهالكك جملة اللحوم كما تشير البيانات الواردة التهات بالجدول رقم (ب) ما بين حد ادني

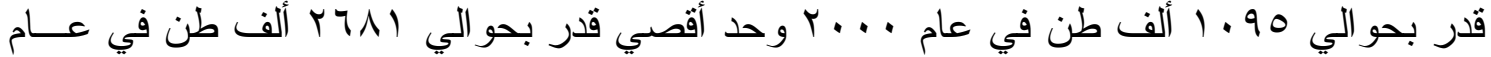
1 10

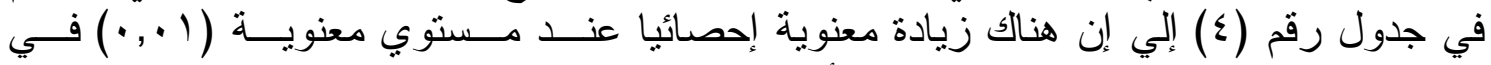

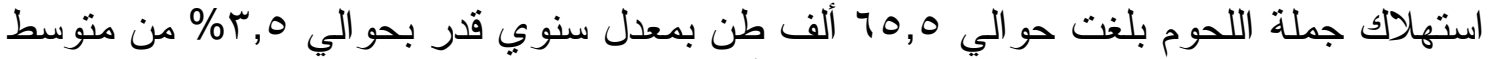

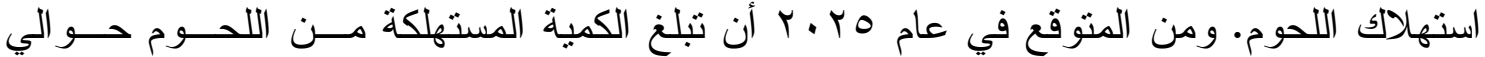

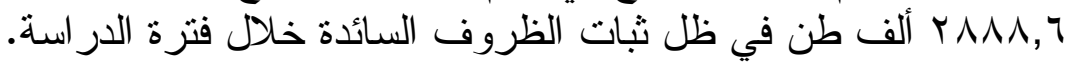




\section{ب - منبة الاكتفاء الذاتي:}

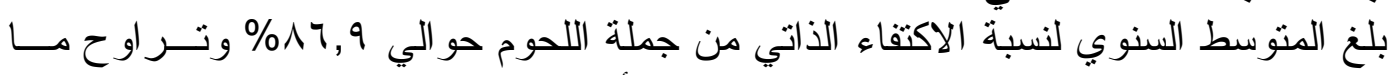

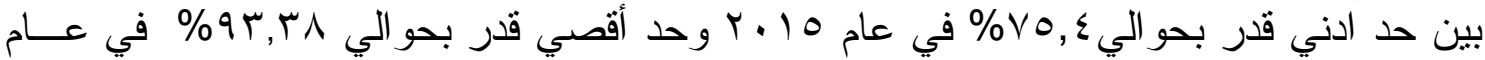

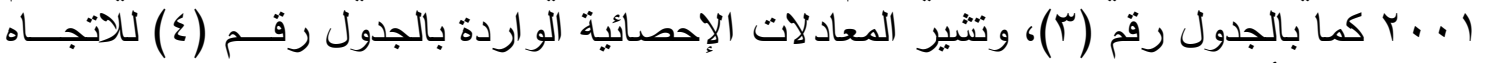

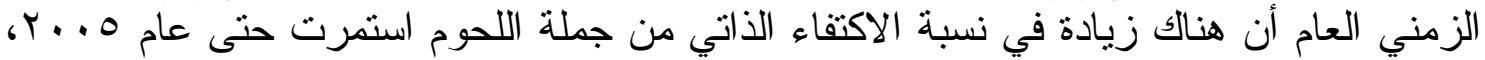

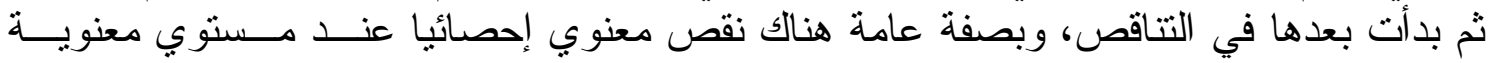

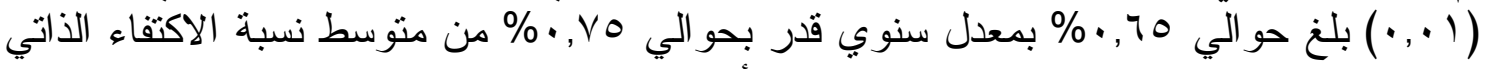

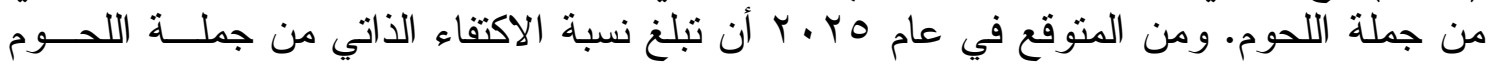

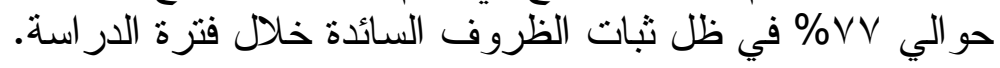

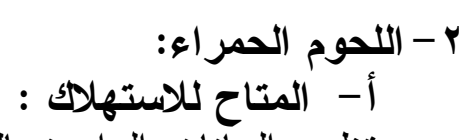

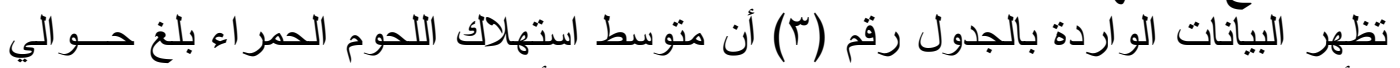

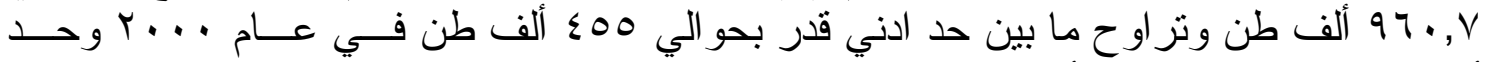

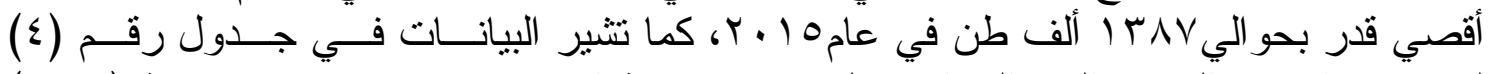

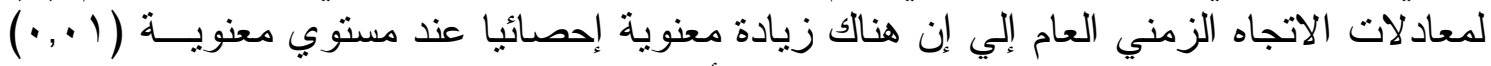

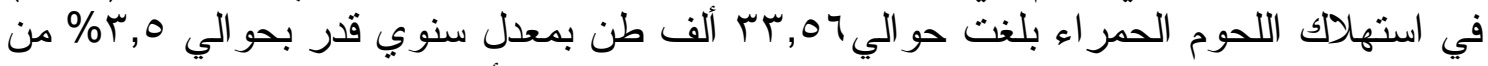

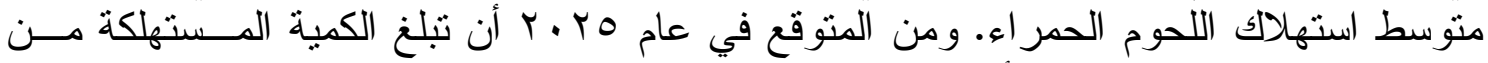

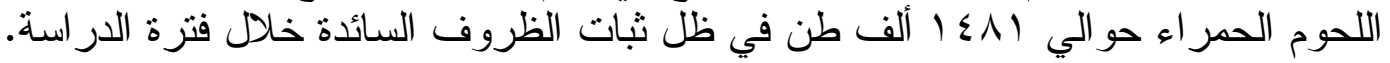

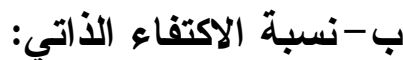

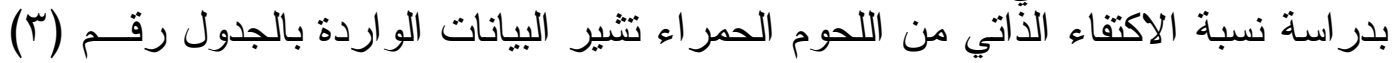

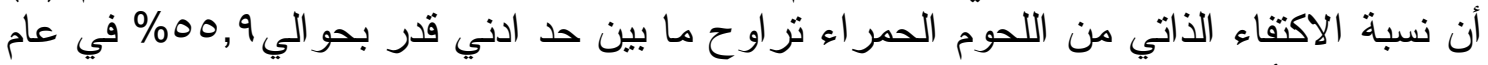

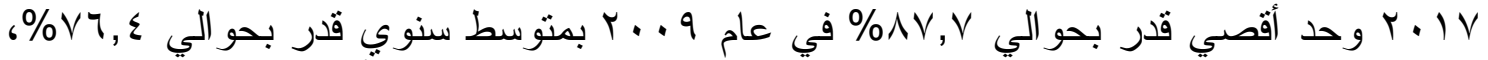

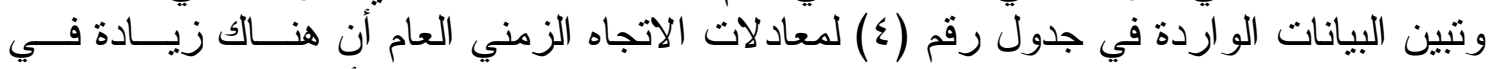

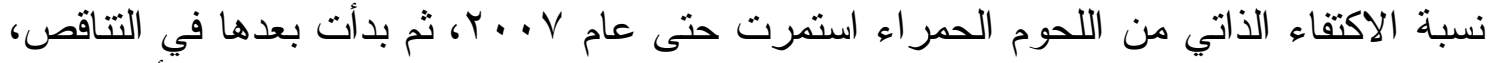

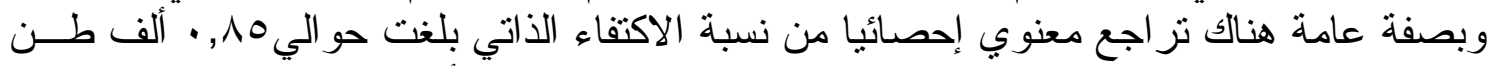

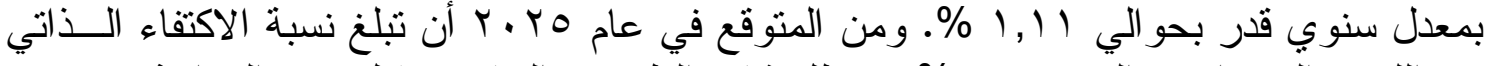

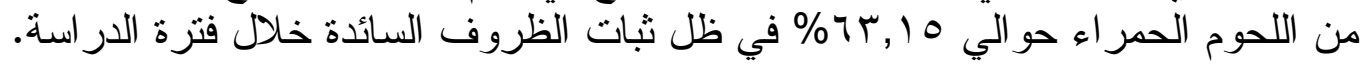


جدول r. تطور المتاح للاستهلاك بالأف طن ونسبة الاكتفاء الأتي لجملة اللحوم والألبان ومنتجاتها

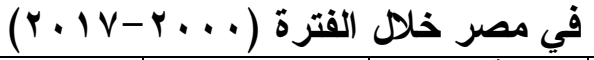

\begin{tabular}{|c|c|c|c|c|c|c|c|c|c|c|c|c|}
\hline \multicolumn{2}{|c|}{ الألبان ومنتجاتها } & \multicolumn{2}{|c|}{ البيض } & \multicolumn{2}{|c|}{ الاسماكت } & \multicolumn{2}{|c|}{ اللحوم البيضاء } & \multicolumn{2}{|c|}{ اللحوم الحمر اء } & \multicolumn{2}{|c|}{ جملة اللحوم. } & \multirow[b]{2}{*}{ السنوات } \\
\hline الاكتفبة & |للاستهلاح & الأكتفاءة & للاستهلاح & |الاكتفاءة & للاستهـلاحك & الاكتفاء & للاستهلاح & الاكتفاءة & للاستهلاحك & نالاكتبة & للاستهلاك & \\
\hline$\vee \wedge, \wedge Y$ & \&70T & $1 \ldots, \ldots$ & rOY & VI,T. & $9 \leq 9$ & $1, \cdot, 4$ & $7 \varepsilon$. & $\pi T, V$ & $\leqslant 00$ & $\wedge \varepsilon, \vee$. & 1.90 & • \\
\hline$v q, r$. & \&NYV & $1, \cdots, \cdots$ & 1 & $V \leq, 71$ & .19 & $\cdots, \wedge$ & 110 & $10, \vee 9$ & VY. & $9 \pi, r \wedge$ & 10\%0 & $\cdots 1$ \\
\hline $1 \cdots, I V$ & 79 & $1 \cdots, \mid V$ & & $\wedge r, \leqslant q$ & Y. & 99,07 & & $1, Y V$ & $\sum V$ & $9 Y, 1$ & ATY & $\cdots r$ \\
\hline Ar,Ir & 7194 & $1 \cdots, r$. & ro. & $\Lambda T, V V$ & $9 \curlyvee \wedge$ & $1 \cdots, \cdot 1$ & $\Lambda Y \wedge$ & $\wedge 7, Y)$ & $9 \cdot 1$ & $9 Y, \wedge r$ & IVTr & $r \ldots r$ \\
\hline ᄉฯ, Yq & & $1 \cdots, r_{0}$ & .9 & V9,7Y & $\cdot \lambda_{7}$ & $\cdots, \cdot 1$ & & $\varepsilon, \leqslant 0$ & & r,AV & $\pi$ Tr. & $\ldots \varepsilon$ \\
\hline$\Lambda V, T \vee$ & $70 \ldots$ & I., r. & $r .9$ & $V 9,7 V$ & 1.17 & $1 \cdot \cdot, r$ & הTI & VV,TV & NoV & $\Lambda \Lambda, T \vee$ & 1711 & $r \ldots o$ \\
\hline 10,11 & $1 \leqslant 9$ & $1 \cdots, 1 \pi$ & & $\Lambda \cdot, \cdot 7$ & 11 & 99,99 & & $\Lambda \cdot, \cdot V$ & $7 \varepsilon$ & $\vee, \wedge r$ & $0 \wedge$. & $r \ldots r$ \\
\hline 94,01 & $r v$ & $1 \ldots, r$ & YQY & $\Lambda Y, r q$ & $Y \leq$ & $9 \wedge, 77$ & 779 & Vr,rV & 97. & $\Lambda Y, V Y$ & 1749 & $r \ldots V$ \\
\hline $1 \cdot 1, \wedge \mathrm{V}$ & $\Lambda \varepsilon$ & $1, \cdot, r$ & 10 & $\checkmark, \wedge 7$ & req & $97, r \leq$ & & $r, \wedge q$ & $9 \leq 7$ & $\Lambda \wedge, \cdot 1$ & $10 \% 7$ & $r \ldots \Lambda$ \\
\hline $1 \cdot 9,7 \leq$ & & $1, \cdot, r$ & ir & $\Lambda V, Y r$ & ror & $97,9 \vee$ & 1 & $\Lambda \vee, \vee \cdot$ & $1 \cdot \varepsilon$ & $91, \cdot v$ & VYO & $r \ldots q$ \\
\hline $110,0 \mathrm{~V}$ & $\$ 997$ & $1 \ldots, 99$ & rVo & $1 V, \leqslant 9$ & 11. & $9 \leq, 9$. & V० & $\Lambda Y, O Y$ & .11 & $\Lambda \vee, \Lambda I$ & $1 \vee 41$ & $r \cdot 1 \cdot$ \\
\hline Ar,TV & & $1 \cdots, .9$ & & $1 \cdot \varepsilon, 1$ & 1.9 & $97, Y Y$ & & $V q, Y v$ & & $\Lambda V, \cdot r$ & & \\
\hline 99,1 . & VY & $1 \cdots, 1$ & 11 & $\Lambda \cdot, \mathrm{V} \cdot$ & $V \cdot$. & $9 r, 0$. & 919 & $V \varepsilon, \varepsilon$ & .94 & $\wedge \bullet, 7$ & 1917 & $r+1 r$ \\
\hline $90, \wedge Y$ & 197 & $|\cdots, r|$ & 10 & $\wedge \vee, q$. & $1 \wedge \cdot V$ & 97,11 & IYTE & $v 7,79$ & $1 . \mathrm{r}$ & $\Lambda V, Y \Lambda$ & YYVY & $r \cdot 1 r$ \\
\hline $91,9$. & 179 & $99,9$. & 17 & $v 0,0$. & 1978 & $9 \vee, \vee 0$ & & 71,10 & 179 & $\varepsilon, r q$ & $r \leq \leq 4$ & $.1 \leq$ \\
\hline$\wedge \wedge, 1$. & $1 r$ & $1 \cdots, 1$ & $Y$ & $V Y, r \cdot$ & 994 & $q r, r \cdot$ & rqr & $\bullet \wedge, \vee$. & rAv & $V \bullet, \varepsilon$. & ४イ & .10 \\
\hline$\wedge q, \wedge$. & 178 & $1 \cdots, 1$ & .9 & $\Lambda \mu, 0$ & 1974 & $9 \leq, V$ & IYNV & $v 7, \cdots$ & 1.74 & $\Lambda \neg, r$. & $r r \leq q$ & $r .17$ \\
\hline $9 r, r$ & $9 \wedge$. & $1 \ldots, 0$ & 1. & $\Lambda 0,1$ & $r .1 Y$ & $90, r$ & & 00,9 & & $v 0,7$ & $r \leq \leqslant$. & $r+I V$ \\
\hline $9 Y, r$ & ovrV, $\varepsilon$ & $1 \cdots, 1 Y$ & PAq & $\Lambda \varepsilon, V$ & $\mid r v \cdot, v$ & $9 \vee, \xi$ & $q 1 Y, q$ & $V 7, \xi$ & $94 \cdot, V$ & $\Lambda 4, q$ & IAVY,V & المنتوسط \\
\hline
\end{tabular}

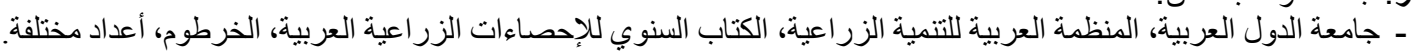

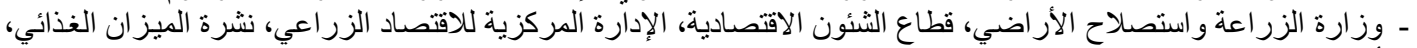
أعداد مختلفة.

\section{r- بملة اللحوم البيضاء

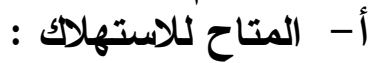

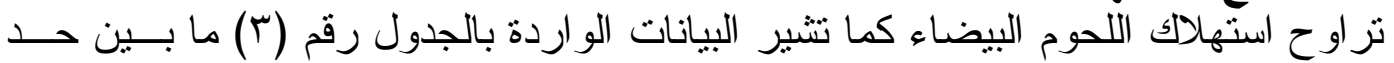

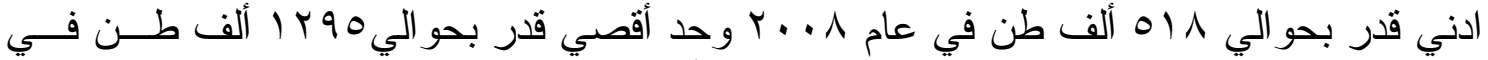

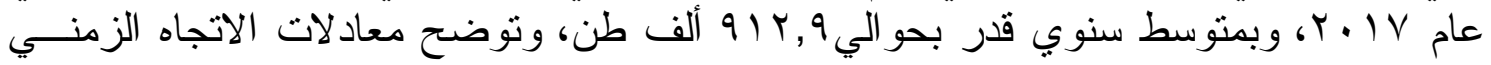

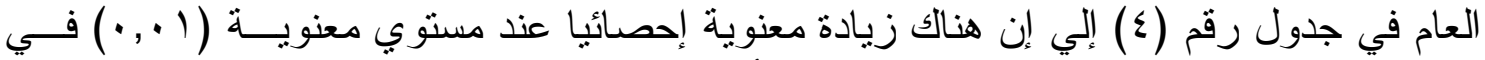

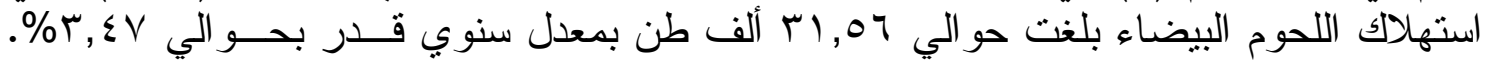

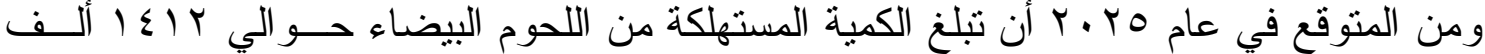

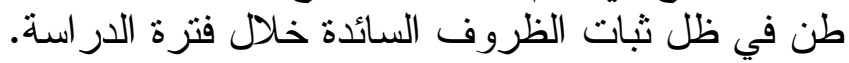

\section{ب-نسبة الاكتفاء الأتي:}

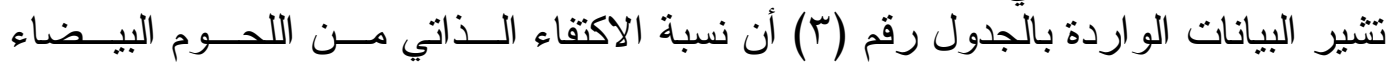

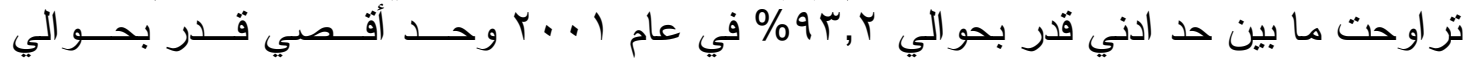

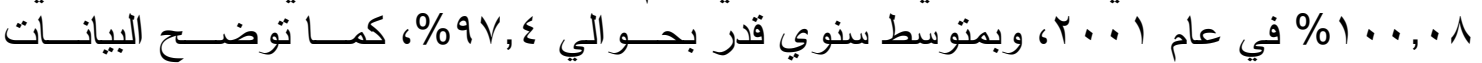

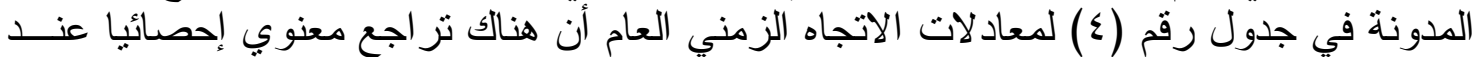

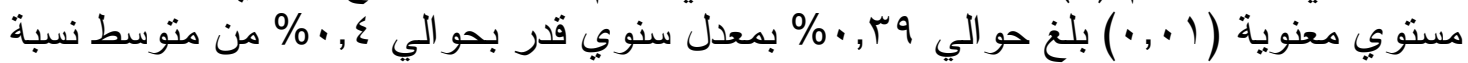

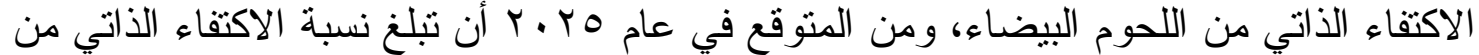

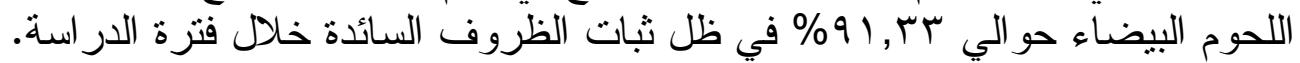


Assiut J. Agric. Sci., 51 (2) 2020 (195-212)

ISSN: 1110-0486

Website:www.aun.edu.eg/faculty_agriculture/journals_issues_form.php E-mail:ajas@aun.edu.eg

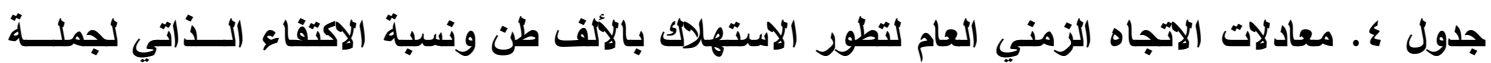

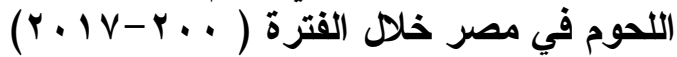

\begin{tabular}{|c|c|c|c|c|c|c|c|}
\hline | المتوقع عام & ف & is & | معدل التغير | & 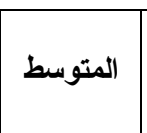 & النموذج & صـ التابع & السلعة \\
\hline 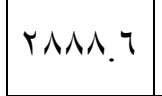 & $* * \varepsilon \leqslant, \wedge$ & $\cdot, \mathrm{V} T \mathrm{~V}$ & r.o & IAVT.V & 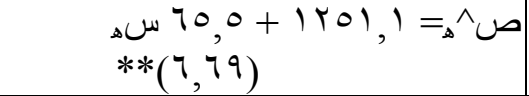 & الاستهلاك & \multirow{2}{*}{ اللخوم } \\
\hline VV & $* * \mid r, \wedge 1$ & $\cdot, \leqslant 7 \pi$ & $\because \vee O_{-}$ & 17.9 & 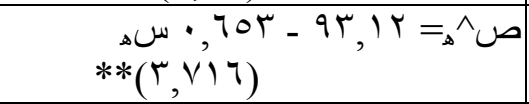 & |نسبة الاكتفاء & \\
\hline $1 \leq \Lambda 1$ & $* * \leqslant \Lambda, 7 \mu$ & $\cdot$, VOr & r.o & $97 . . V$ & 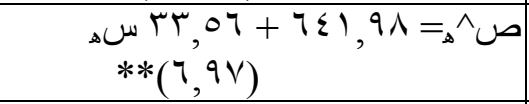 & الاستهلاك & \multirow{2}{*}{ الحمراء } \\
\hline & $* . \wedge . \wedge 0$ & $\cdot, r r r$ & $1.11-$ & $V 7 . \varepsilon$ & 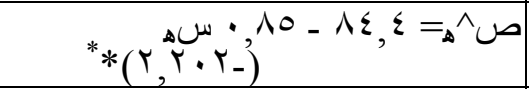 & |نسبة الاكتفاء & \\
\hline $1 \leq 1 r$ & $* * \mid r, \wedge$ & $\cdot, \leqslant \mu$ & $r . \varepsilon V$ & $q 1 Y . q$ & 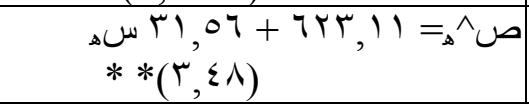 & الاستهالك & \multirow{2}{*}{ البيضاء - اللدوم } \\
\hline חז. 91 & $* * \varepsilon 1,10$ & $\cdot, V Y$. & $\cdot \varepsilon_{-}$ & $9 \vee . \varepsilon$ & 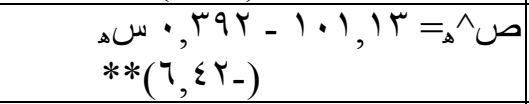 & |نسبة الاكتفاء & \\
\hline$r \leqslant V r$ & $* * q \vee, \leqslant r$ & $\cdot, 109$ & 0.19 & $\mid r v \cdot . v$ & 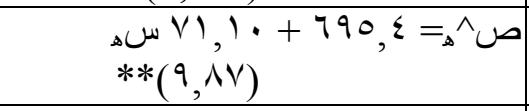 & الاستهلالك & \multirow{2}{*}{ الاسماك } \\
\hline- & $\cdot, \wedge \varepsilon$ & $\cdot, 0$ & - & $\Lambda \varepsilon . \vee$ & $\begin{array}{l}\Delta \omega \cdot, \xi \xi+\Lambda \cdot, 0={ }_{\Delta}^{\wedge} ص \\
(\cdot, q r)\end{array}$ & |نسبة الاكتفاء & \\
\hline- & $\cdot, \leq \leqslant 9$ & $\cdot, r V$ & - & r^q & 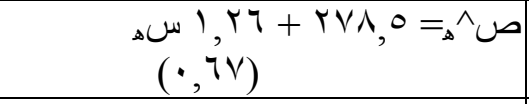 & الاستهلالك & \multirow{2}{*}{ البيض } \\
\hline- & ד & $\cdot, \cdot r V$ & - & $1 \cdots .14$ & 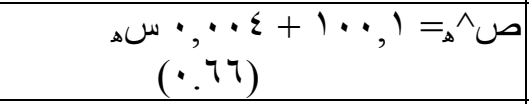 & |نسبة الاكتفاء & \\
\hline- & $r, \cdot 1$ & $\cdot, 114$ & - & OVMV. $\varepsilon$ & 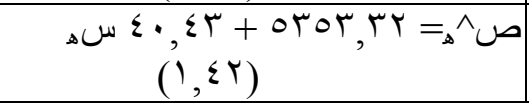 & الاستهلاك & \multirow{2}{*}{ 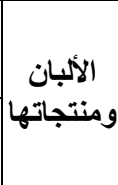 } \\
\hline $1.1 . r$ & $* r, V \backslash 0$ & | ו & אד. & $9 Y . r$ & 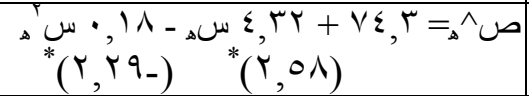 & |نسبة الاكتفاء & \\
\hline
\end{tabular}

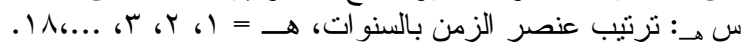

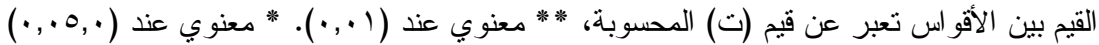

المصدر: حسبت من بيانات الجدول رقم (r).

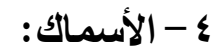

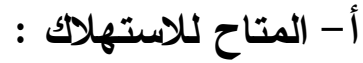

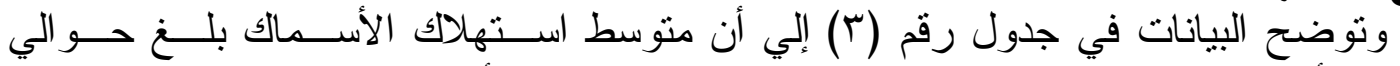

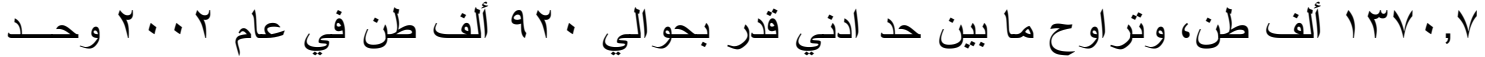

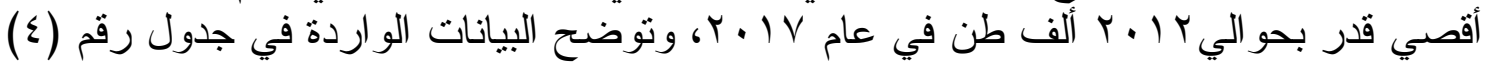

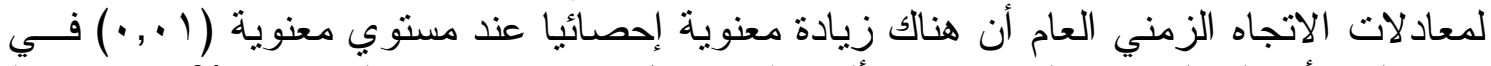

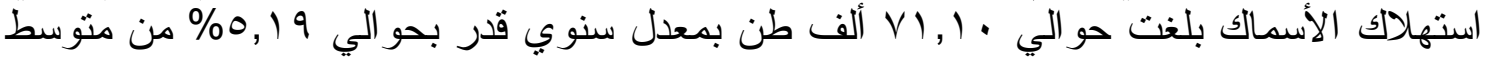

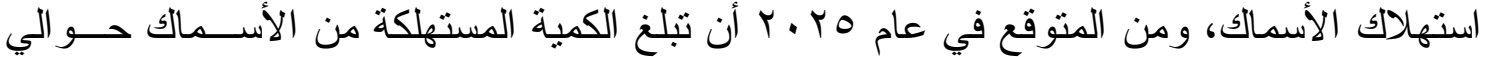

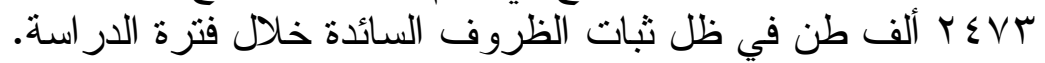

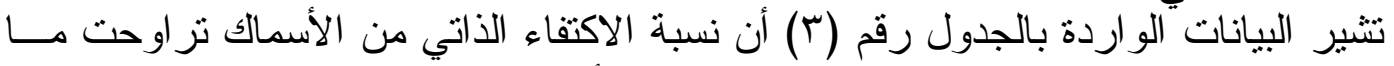

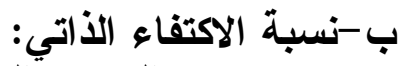

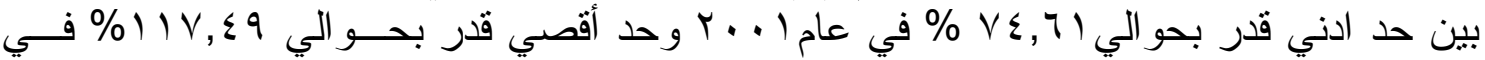




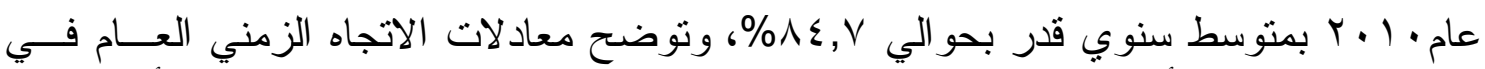
جدول رقم (ع) إلي أن هناك زيادة غير فئ معنوية إحصائيا في نسبة الاكتقاء الذاتي من الأسماك.

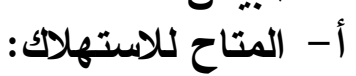

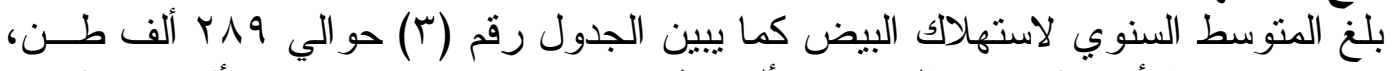

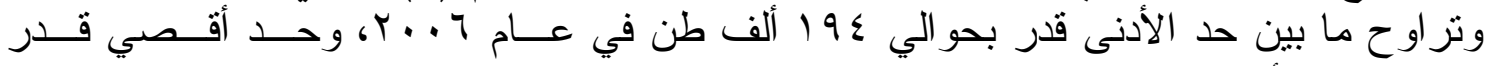

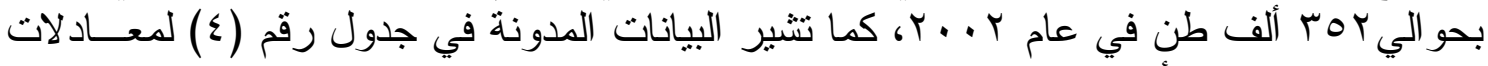

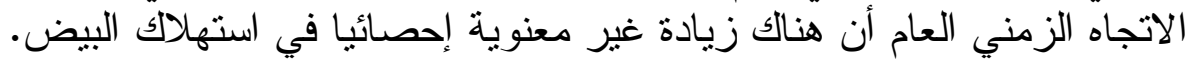

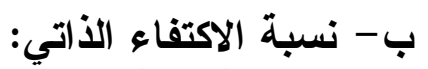

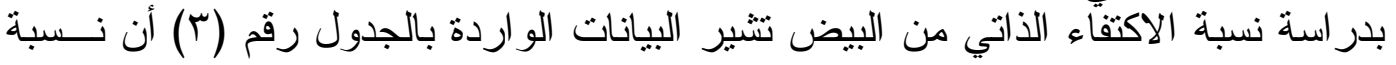

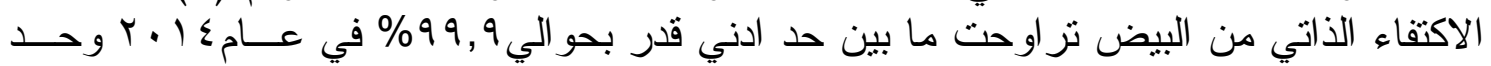

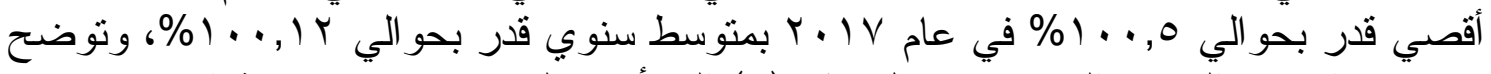

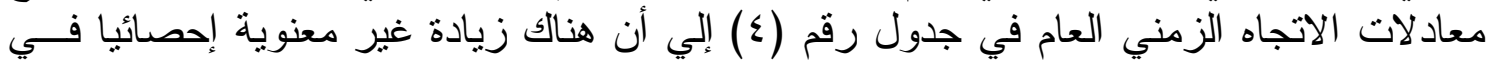

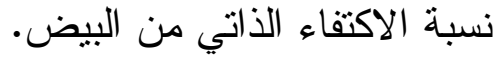

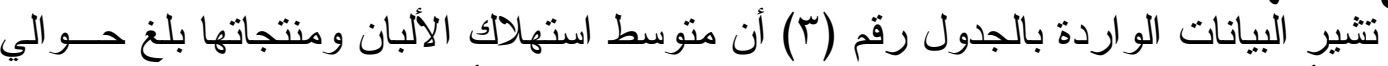

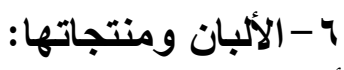

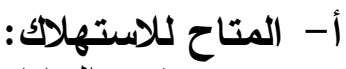

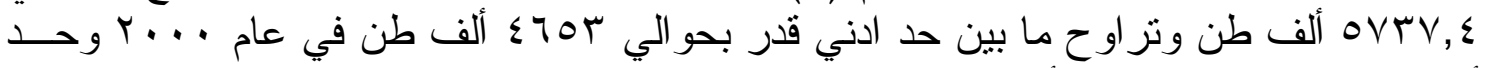

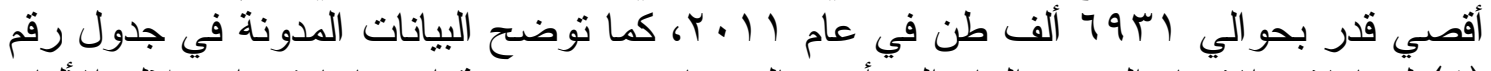

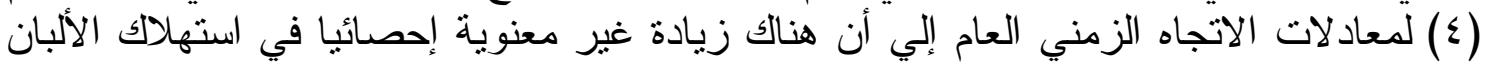

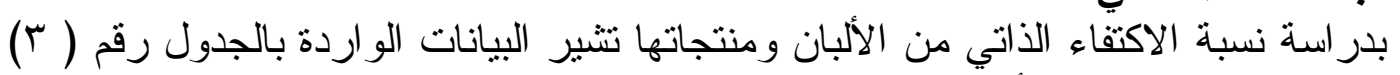

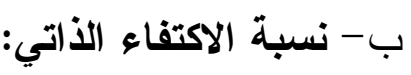

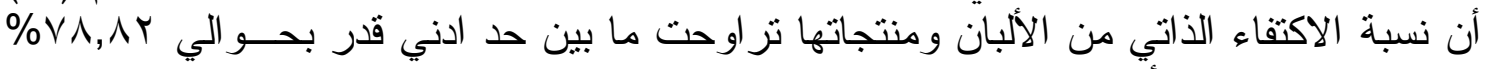

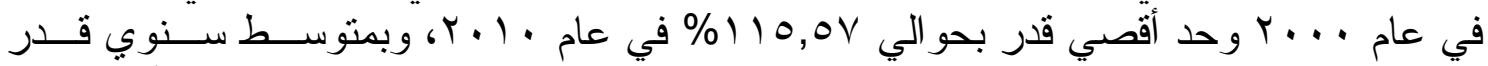

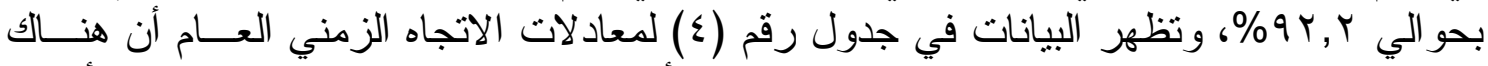

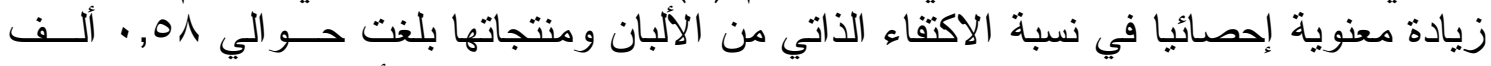

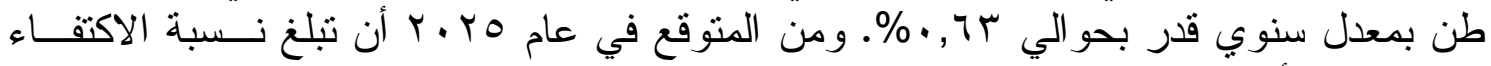

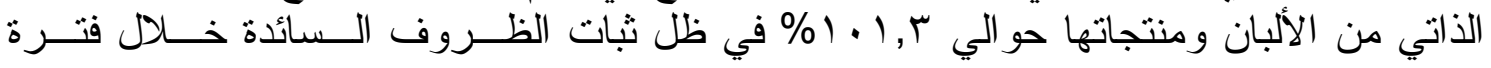

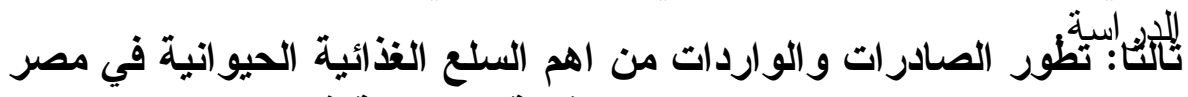

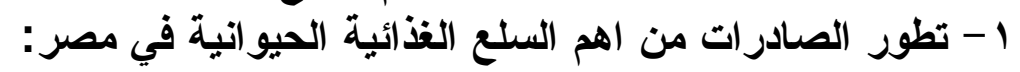

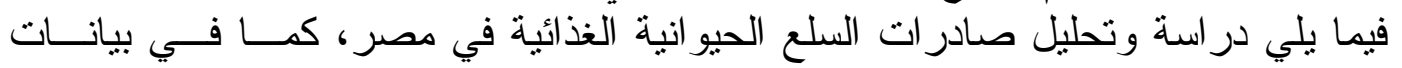

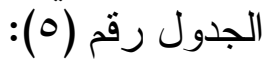

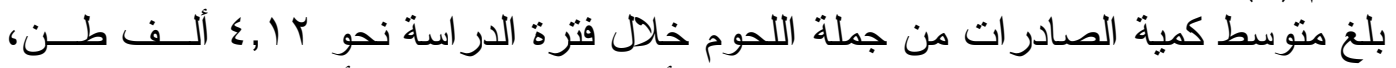

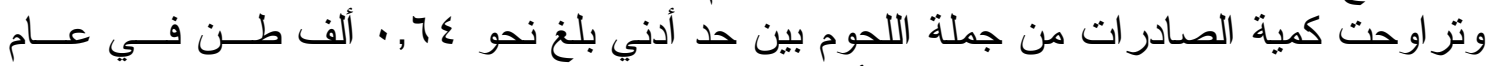

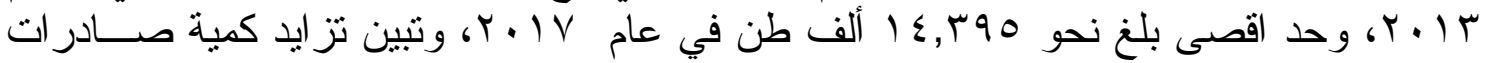

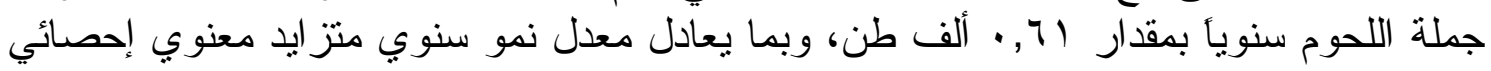

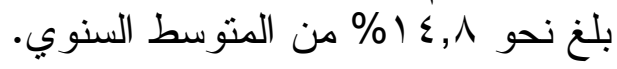




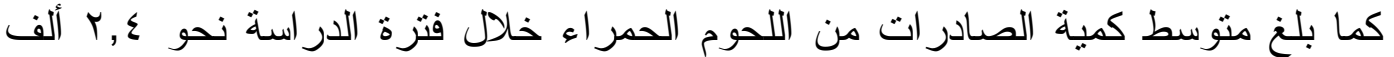

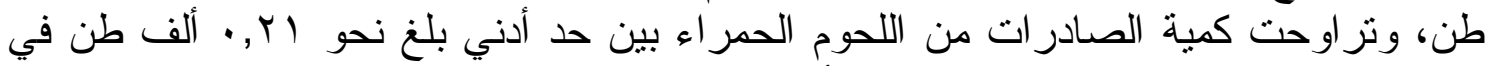

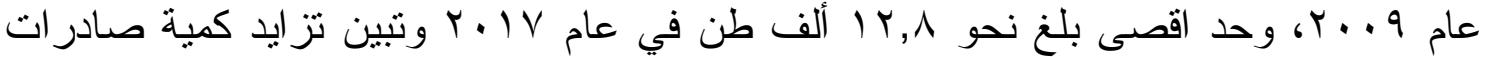

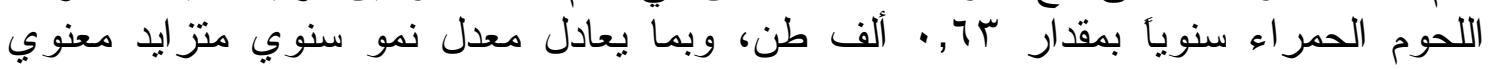

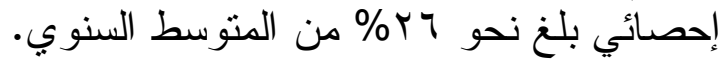

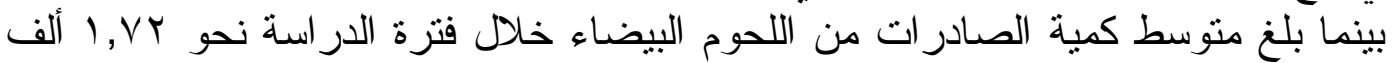

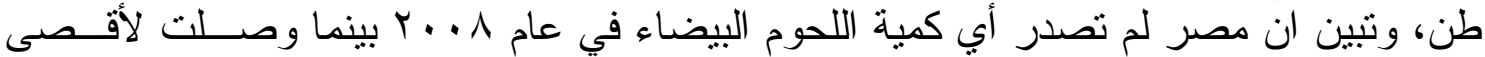

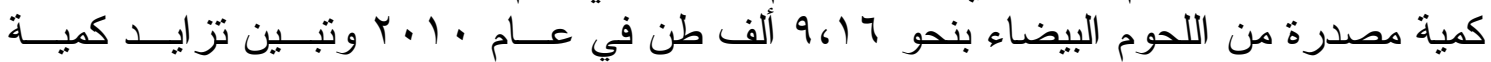

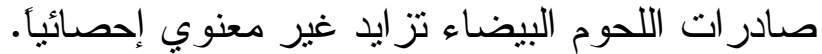

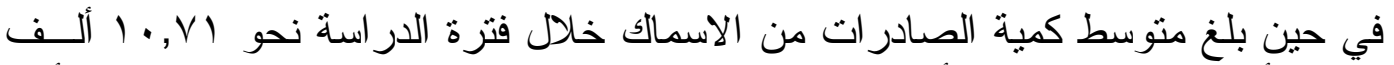

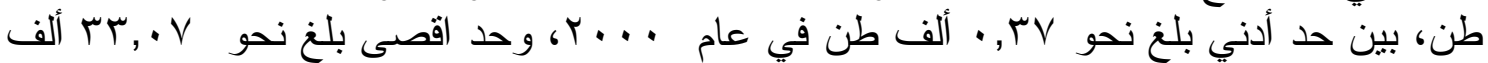

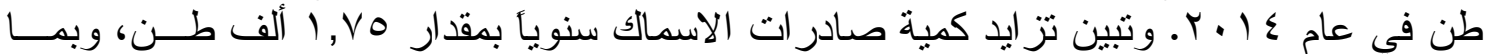

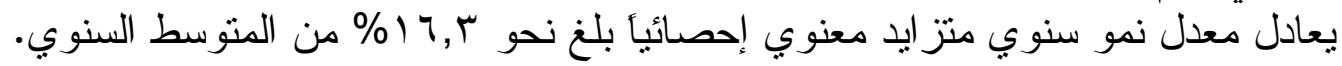

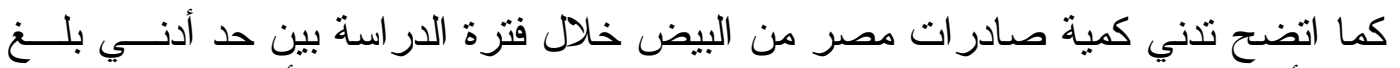

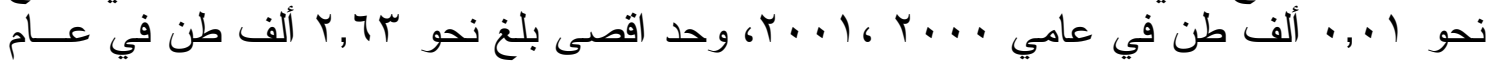

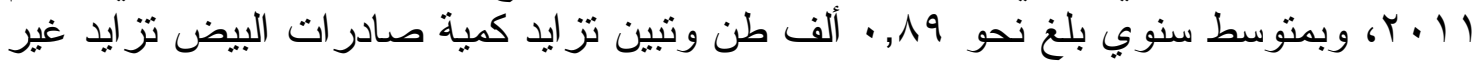
معنوي إحصائي.

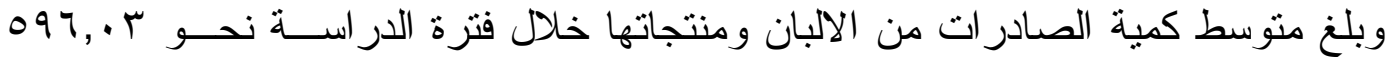

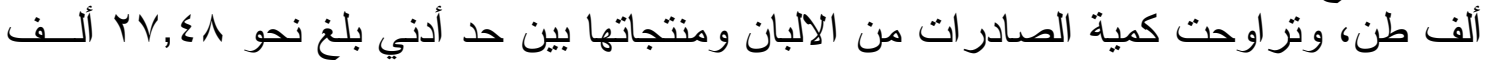

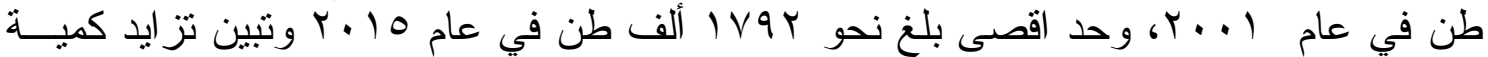

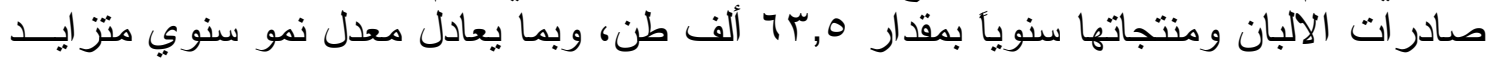
معنوي إحصائياً بلغ نحو 7, • (1\% من المنوسط السنوي.

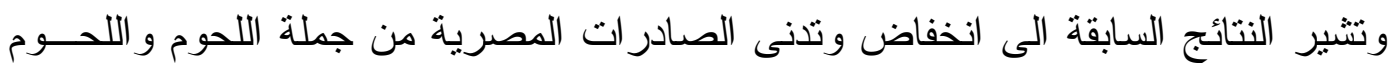

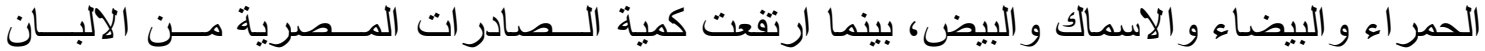

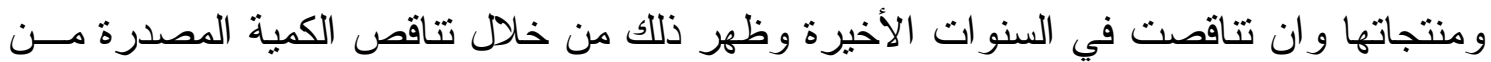
الالبان ومنتجاتها خلال فترة الدر اسة.

\section{r-نطور الواردات من اهم السلع الغذائية الحيو انية في مصر :}

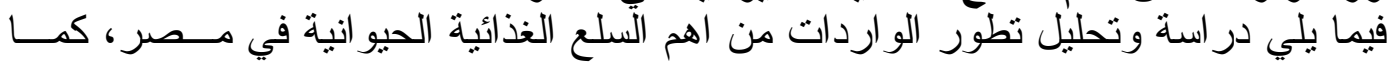

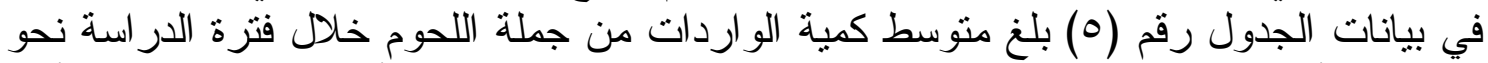

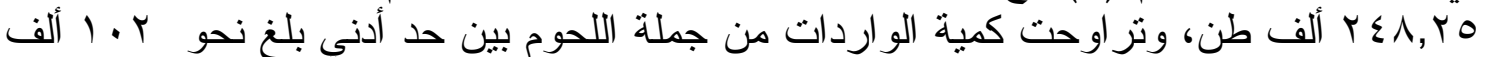

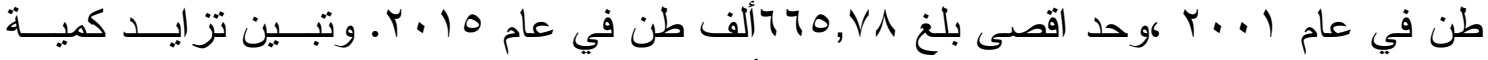

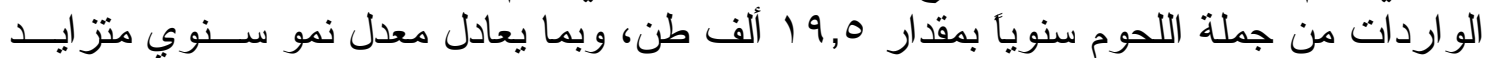

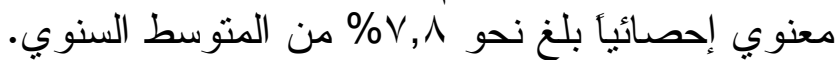

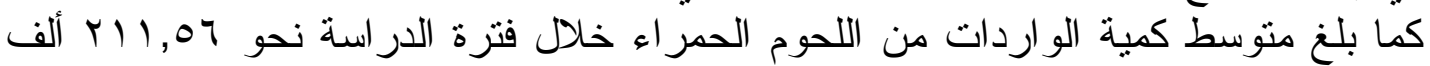

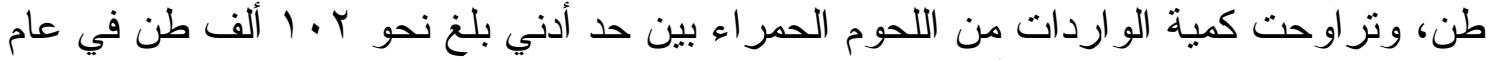

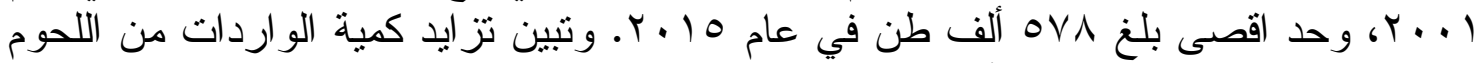

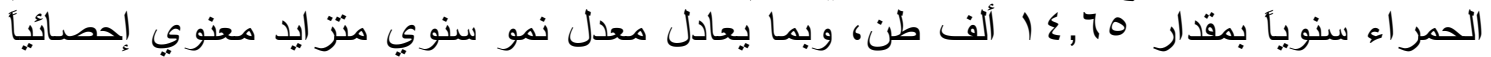

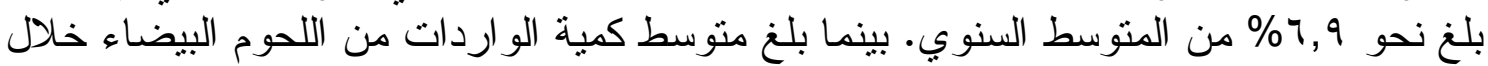

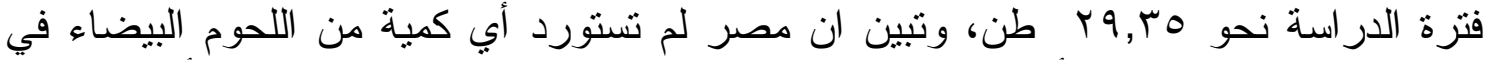

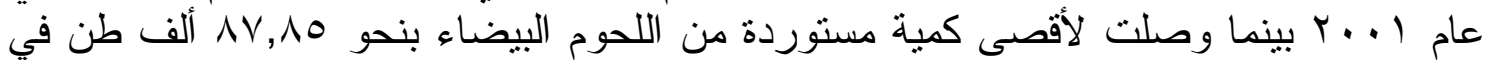




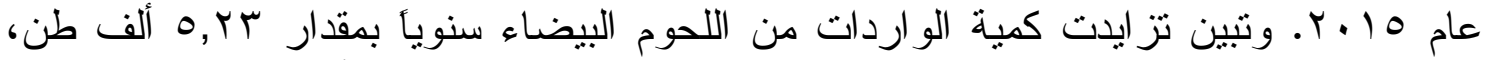

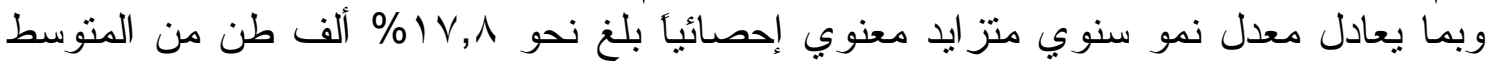
السنوي.

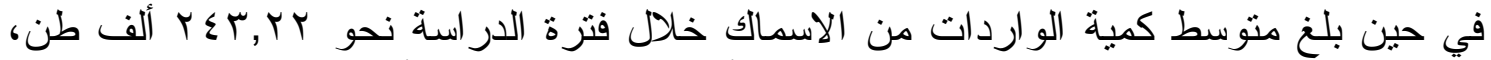

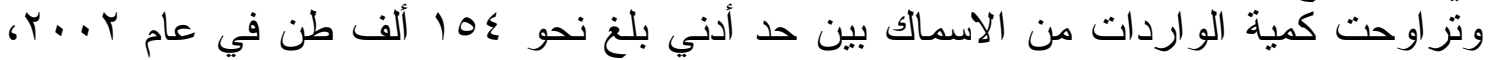

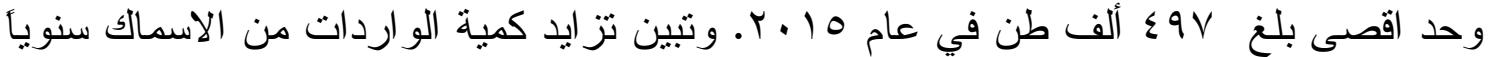

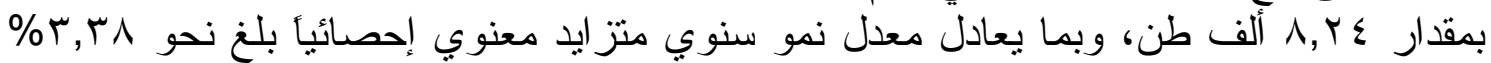
من المتوسط السنوي.

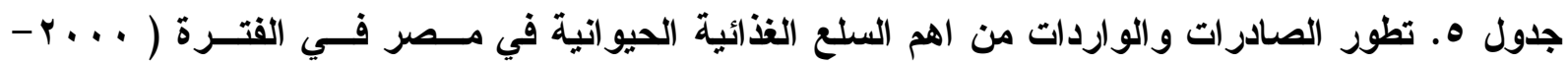

\begin{tabular}{|c|c|c|c|c|c|c|c|c|c|c|c|c|}
\hline \multicolumn{2}{|c|}{ الألبان ومنتجاتها } & \multicolumn{2}{|c|}{ البيض } & \multicolumn{2}{|c|}{ الاسماك } & \multicolumn{2}{|c|}{ اللحوم البيضاء } & \multicolumn{2}{|c|}{ اللحوم الحمر اء } & \multicolumn{2}{|c|}{ اجملة)/للحوم V } & \multirow{3}{*}{ السنوات } \\
\hline كمية & كمية & كمية & كمية & كمية & كمية & كمية & كمية & كمية & كمية & كمية & كمية & \\
\hline الواردات & الصادرات & الواردات & الصادرات & الواردات & الصادرات & الواردات & الصادرات & الواردات & الصادرات & الواردات & الصادرات & \\
\hline $1 . Y_{0}$ & $\varepsilon \cdot, \mid r$ & $\cdot, \cdot 1$ & $\cdot, \cdot 1$ & rro & $\cdot, r V$ & $\cdot, \cdot 1$ & $\cdot, r q$ & Ir. & $\cdot, \varepsilon \Gamma$ & 171 & $\cdot, \Lambda Y$ & $r \ldots$ \\
\hline $1 . T r$ & $T V, \Sigma \Lambda$ &., .1 & $\cdot, \cdot 1$ & T7T & $1, \leqslant 7$ & $\cdot, \ldots$ & $\cdot, 7 \varepsilon$ & $1 \cdot T$ & $\cdot, \Gamma \xi$ & $1 \cdot Y$ & $\cdot, 91$ & $r \ldots 1$ \\
\hline 1110 & Yq,६। &., .1 &., 09 & $10 \leqslant$ & T,OT & $0, \pi 4$ & . ז. & $1 \varepsilon$. & $\cdot, \leqslant \xi$ & $1 \leqslant 0$ & $1, \cdot \varepsilon$ & T.. T \\
\hline $1.9 \mathrm{~V}$ & $01, \wedge \varepsilon$ &., .1 & 1,10 & $17 \pi$ & $r, 1 r$ & $\cdot, .9$ & $\cdot, \mathrm{V} \leqslant$ & TrT & $\cdot, \mathrm{VY}$ & TrT & $1, \leqslant 7$ & $r \ldots r$ \\
\hline Vos & $07, \times 1$ & $\cdot, \ldots$ & $r, \cdot A$ & TYT & $0, \cdot Y$ & $\cdot, \leqslant V$ & $\cdot, 0 \mathrm{~V}$ & 117 & $\cdot, \wedge$. & $11 \mathrm{~V}$ & $1, \pi V$ & $r \ldots \varepsilon$ \\
\hline 904 & $101, \ldots$ &., .0 & 1,04 & TYT & $0,1 Y$ & $\cdot, 1 \leqslant$ & • & 1.9 & $\cdot, 7 \leqslant$ & 194 & $\cdot, 91$ & r... \\
\hline $11 \% \mathrm{r}$ & $1 \leq r, \cdots$ & $\cdot, .9$ & $\cdot, \Gamma$ & rol & $\varepsilon, r V$ & $\cdot, \mathrm{r} \cdot$ & $\cdot, 11$ & 194 & $\cdot, \mathrm{V}$ & 195 & $\cdot, \Lambda Y$ & 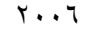 \\
\hline $9 . r$ & $79,0 Y$ & $\cdot, \cdots$ &., .0 & ros & $\varepsilon, \varepsilon Y$ & $9,0 \mathrm{~V}$ &., 00 & rq9 & 1,00 & TVA & 1,1 & $r \ldots V$ \\
\hline 101 & $97 \Lambda, \ldots$ & $\cdot, 19$ & $\cdot, Y \uparrow$ & 171 & $7, r V$ & YI,Yo & $\cdot$, & $17 \varepsilon$ & $r, V T$ & 1117 & $r, V \mu$ & $r \cdots A$ \\
\hline$\sum \vee 7$ & $11 V \cdot, \ldots$ & $\cdot, \mathrm{IV}$ & $\cdot, \cdot 1$ & 171 & V,VO & rr,7q & $\varepsilon, 0 \wedge$ & 147 & $\cdot, Y I$ & 109 & $\varepsilon, \vee q$ & $r \ldots q$ \\
\hline$T .10$ & IVqr,.. &., 10 & $\cdot, \S 1$ & IAY & $r, V\urcorner$ & $\sum V, T Y$ & 9,17 & $T V A$ & $1, \leqslant V$ & TYT & $1 \cdot, 7 \pi$ & $r \cdot 1 \cdot$ \\
\hline$r 7 \leq 9$ & $101 \mathrm{~V},$. & $\cdot, .7$ & r, & IVA & $r, r)$ & $r \leqslant, \wedge q$ & r, T, & YIT & $\cdot, 9$. & $T \leq V$ & $r, Y \tau$ & $r+11$ \\
\hline $9 \vee 7$ & 9Y1,.. & $\cdot, 7$ & $\cdot, 0$ & TYE & $\mid r, 1 \cdot$ & $7 \cdot, 9$. & $\cdot, \wedge$ & YוA & $r, Y \cdot$ & TVq & $r, \ldots$ & $T \cdot I T$ \\
\hline 1107 & $111 \pi, \ldots$ & $\cdot, \uparrow$ & . & THT & $I V, \ldots$ & $\leqslant \vee, 0$. & $\cdot, \sum Y$ & $T \leqslant Y$ & $\cdot, Y Y$ & $r \wedge q$ & $\cdot, 7 \varepsilon$ & $T .1 T$ \\
\hline $1 \leq 71$ & $970,0 \mathrm{~V}$ & $1, r \cdot$ & $1,1 Y$ & T & $r \mu, \cdot V$ & $r 4, \cdots$ & 7,04 & ror &., 07 & rq. & $8, .9$ & $r \cdot 1 \leqslant$ \\
\hline $1 \times 9$ & orq,r. & $\cdot, 7 \wedge$ & $1 \cdot, \cdot 1$ & $\sum 9 V$ & YY,VA & $\wedge \vee, \wedge \circ$ & $\cdot, 0$ & $0 V \lambda$ & 0,1 . & 777 & 0,09 & $r .10$ \\
\hline $110 \mathrm{~V}$ & $01 \cdot, 10$ & $\cdot, 0 \leqslant$ & $\cdot, \Lambda Y$ & ror & rᄉ, Vo & $v_{\cdot}, r_{0}$ & $1,0 \varepsilon$ & rTV & 11,97 & או ז & $1 \%, 0$ & $r .17$ \\
\hline IYTO & $09 \leqslant, 0$ &., $\mathrm{VO}$ & $\cdot, \wedge 9$ & rVO & $r Y, O$ & $\Lambda Y, 0 T$ & 1,09 & $r \wedge 0$ & $1 Y, \Lambda$ & rTV,07 & $1 \leq, r q$ & $r . \mid V$ \\
\hline $11 r 9,1$ & $097, .4$ & $\cdot, r^{9}$ & I,TY & $T \leqslant Y, Y Y$ & $1 \cdot, V \mid$ & rq,ro & $1, V Y$ & $\bar{Y} \backslash 1, T$ & Y,. & $r \leqslant \Lambda, Y_{O}$ & $\varepsilon, I r$ & المتوسط \\
\hline
\end{tabular}

- جامعة الدول العربية، المنظمة العربية للتتمية الزر اعبة، الكتاب السنوي للإحصاءات الزر اعية العربية، الخرطوم، أعداد مختلفة. - - الجهاز المركزي للتعبئة العامة و الإحصاء، نشرة التجارة الخارجية، أعداد متفرقة .

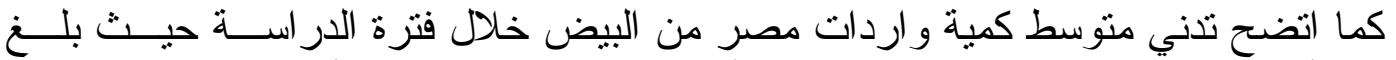

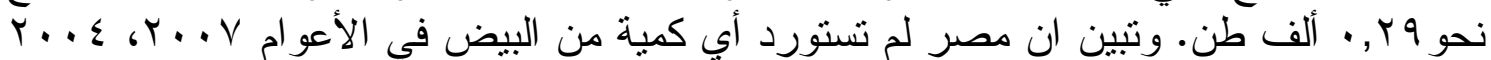

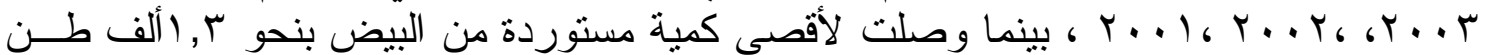

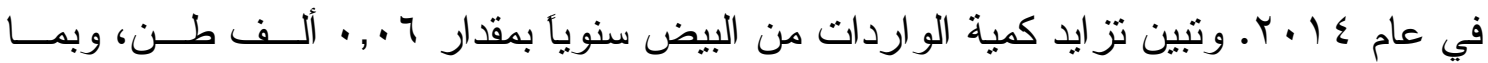

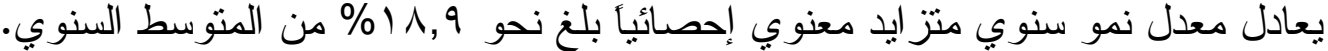


Assiut J. Agric. Sci., 51 (2) 2020 (195-212)

ISSN: 1110-0486

Website:www.aun.edu.eg/faculty_agriculture/journals_issues_form.php E-mail:ajas@aun.edu.eg

جدول ج. معادلات الاتجاه الزمني العام الصادرات والواردات من اهم السلع الغذائية الحيوانية مــر

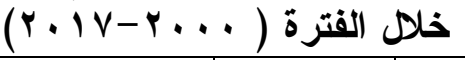

\begin{tabular}{|c|c|c|c|c|c|c|}
\hline ف & זנ & \begin{tabular}{|l|} 
السنول التغير \\
\%
\end{tabular} & المتوسط & النموذج & المتغير التابع & السلعة \\
\hline$* *|\wedge| \leq$, & .,041 & $1 \leqslant .1$ & $\varepsilon .1 T$ & 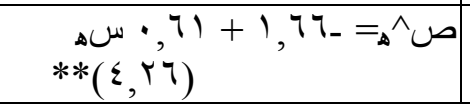 & صادرات & \multirow{2}{*}{ جملة اللحوم } \\
\hline **rr, &., 094 & $\vee .10$ & $r \leqslant \Lambda . r_{0}$ & $\begin{array}{l}\left.\operatorname{su}_{* *(\varepsilon, \wedge} 19,0+r\right) \\
* r, \varepsilon r={ }_{\Delta} \wedge \\
\end{array}$ & الواردات & \\
\hline$* * 11,0$ r & $\cdot, \leqslant 19$ & ry. ro & $r_{.} \varepsilon$. & 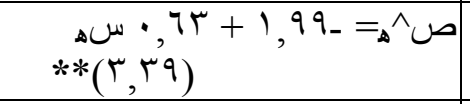 & صادرات & \multirow{2}{*}{ ال الحمراء - الحوم } \\
\hline$* * 1 \leqslant, 7 \pi$ & $\cdot, \leqslant \wedge$ & 7.91 & r11.07 & 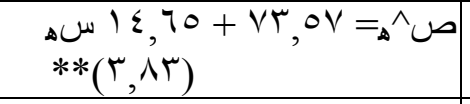 & الواردات & \\
\hline- &,, 97 & - & $1 . V r$ & 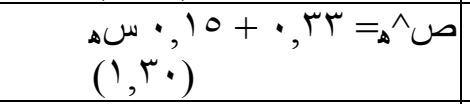 & صادرات & \multirow{2}{*}{ البيضاء - الليو } \\
\hline$* * \wedge 1, \cdot r$ & $\cdot, \wedge \varepsilon$ & $1 V . A$ & rq. \%० & 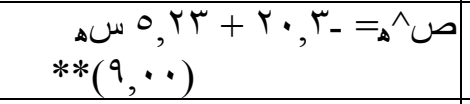 & الواردات & \\
\hline$* * \mu \wedge, T)$ & $\cdot, v \cdot v$ & $17 . r \varepsilon$ & $1 \cdot . v 1$ & 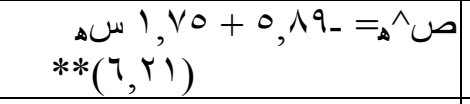 & صادر ات & \multirow{2}{*}{ الاسماك } \\
\hline$* 0,91$ & $\cdot, r V$ & T. ז & $r \leqslant T . Y T$ & $\begin{array}{l}\Delta \cup \wedge, Y \xi+\mid T \leqslant, q r=\wedge \\
*(r, \leqslant 0)\end{array}$ & الواردات & \\
\hline- & $\cdot, 1 \cdot r$ & - & I.r & 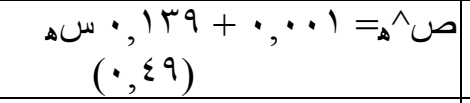 & صادرات & \multirow{2}{*}{ البيض } \\
\hline$* * r \uparrow, 79$ & D & 11.9 & $\cdot r q$ & 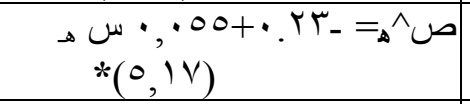 & الواردات & \\
\hline$* * \wedge, \wedge т$ & ד & $1 . .70$ & $097 .+r$ & 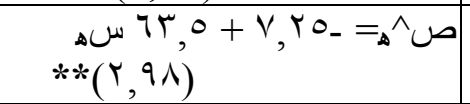 & صادر ات & \multirow{2}{*}{ 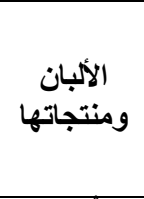 } \\
\hline- & $\cdot 1$ & - & $11 \times 9.1$ & 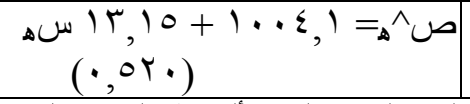 & الواردات & \\
\hline
\end{tabular}

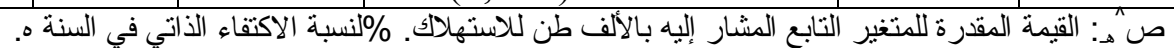

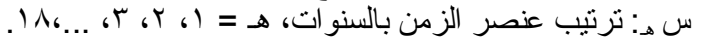

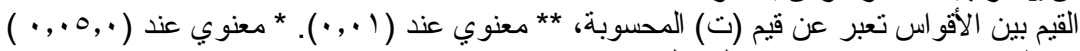

المصدر: حسبت من بيانات الجدول رقم (0).

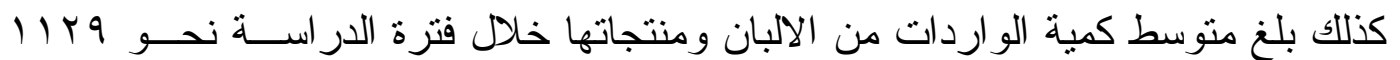

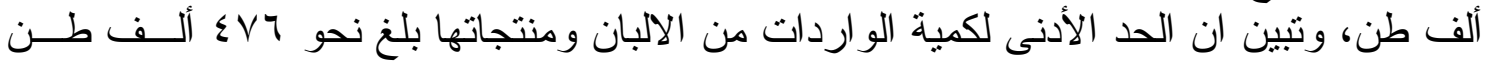

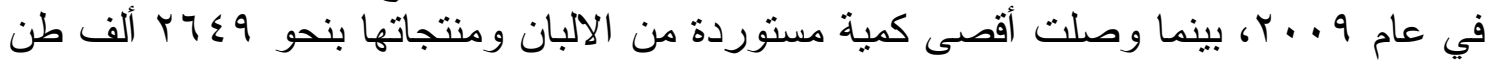

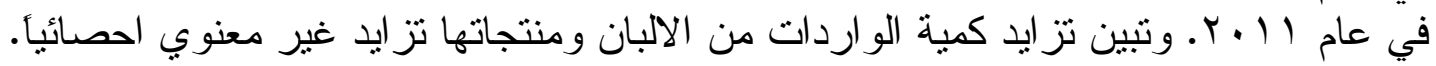

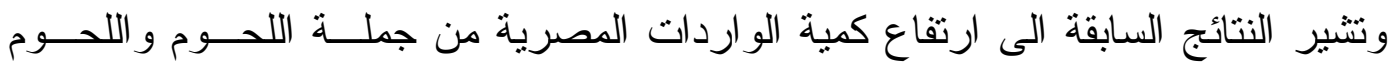

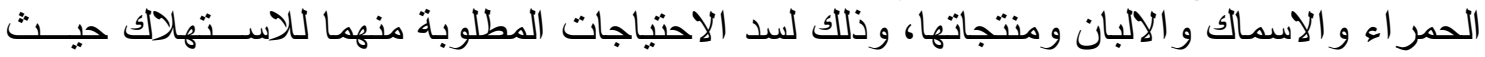

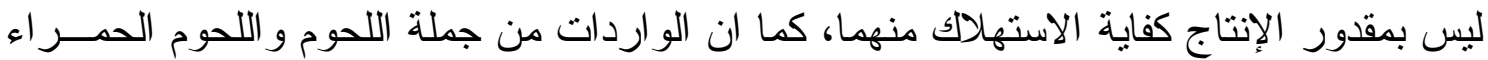

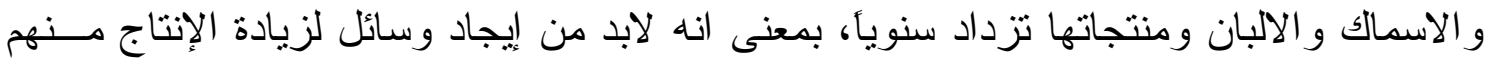

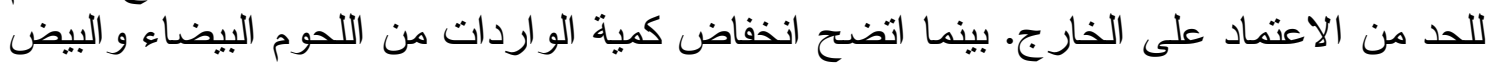

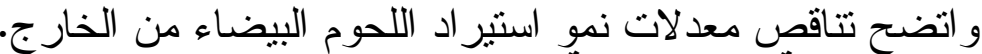

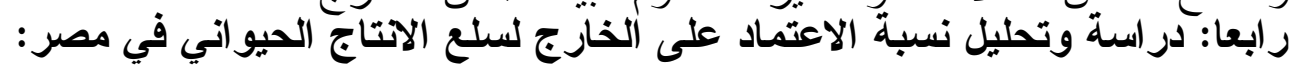

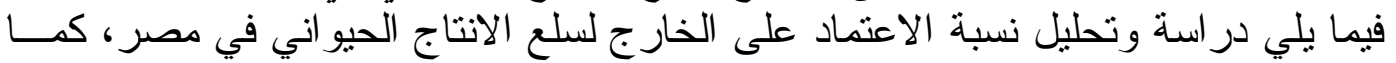
في بيانات الجدول رقم (V): 


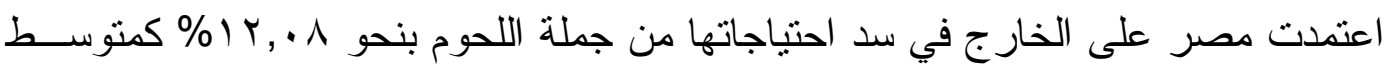

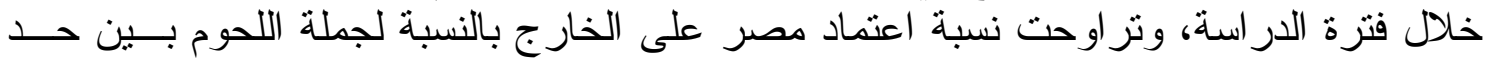

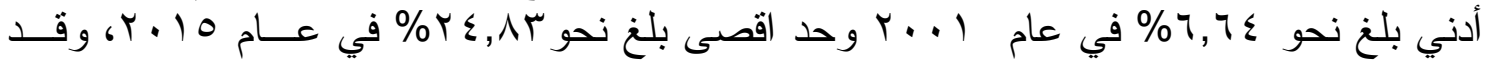

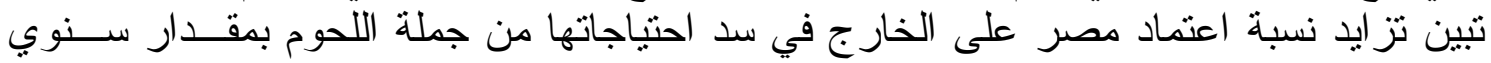

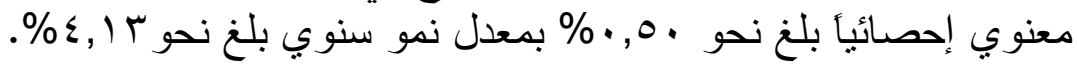

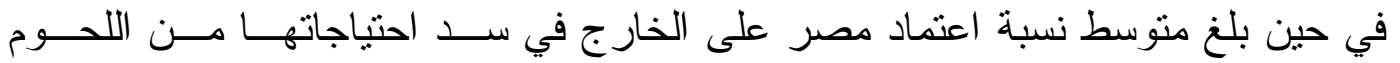

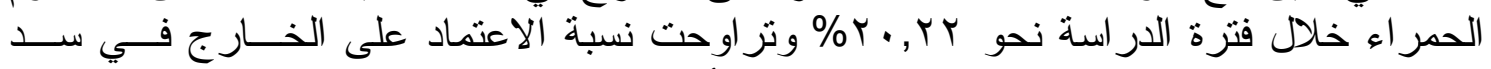

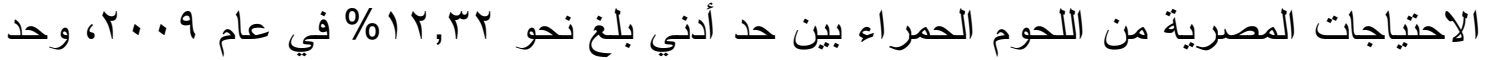

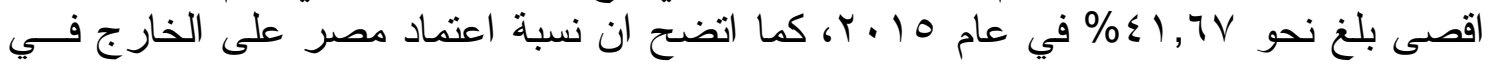

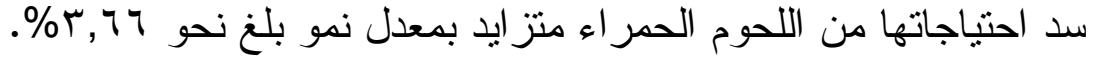

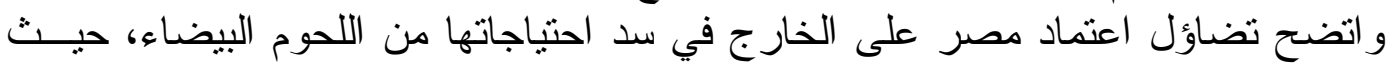

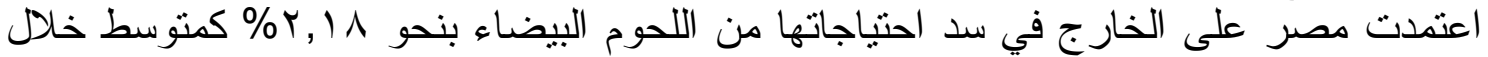

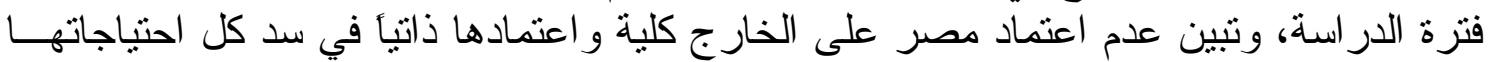

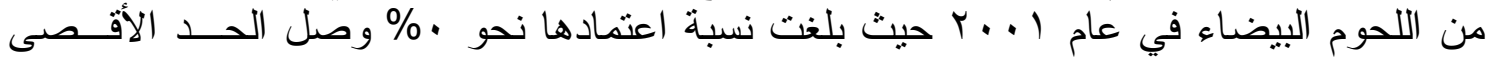

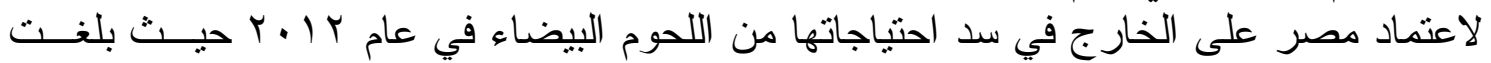

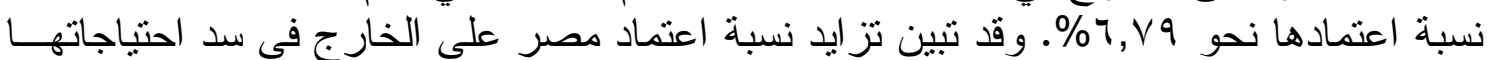

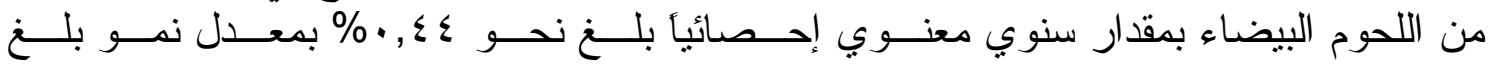

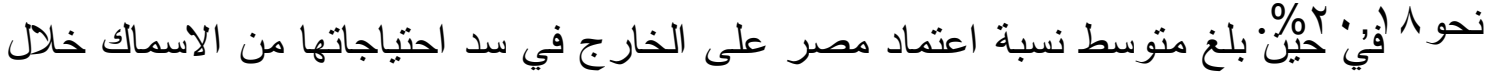

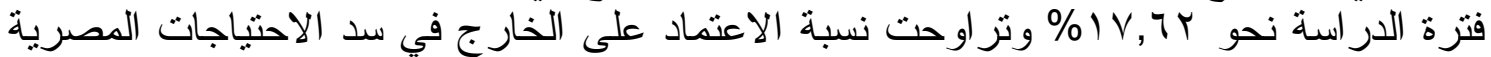

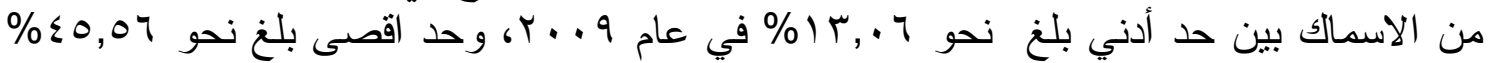

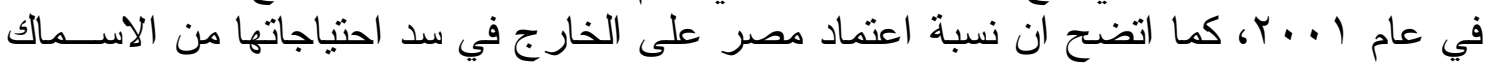

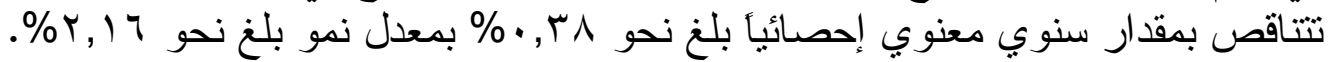

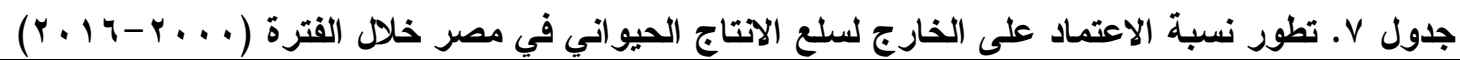

\begin{tabular}{|c|c|c|c|c|c|c|}
\hline الألبان ومنتجاتها & البيض & الاسمـاك & اللحوم البيضاء & اللحوم الحمراء & جملة اللحوم & السنوات \\
\hline$r,, \cdot r$ & $\theta, \cdots \varepsilon$ & $r r, Y l$ & $\cdot, \cdots r$ & $r \neg, r V$ & $10, r \leqslant$ & r... \\
\hline rI, r & $\cdot, \cdots \varepsilon$ & Y0,07 & $\cdot, \cdot$ & $1 \varepsilon, 1 V$ & $7,7 \leqslant$ & r... \\
\hline$Y I, 0 V$ &,$\cdots r$ & $17, \leqslant V$ &.,$\leqslant 9 \leqslant$ & $\overline{1 \Lambda}, V \varepsilon$ & $v, q 1$ & $r \ldots r$ \\
\hline$|v, V|$ & $\cdot, \cdots r$ & $1 V, 07$ & $\cdot, \cdot 11$ & $1 T, \wedge \Lambda$ & $V, Y T$ & $r \ldots r$ \\
\hline$|\varepsilon, \wedge|$ & $\cdot, \cdot \cdot$ & $r \cdot, \lambda)$ & $\cdot, \cdot 0 \leqslant$ & 10,09 & V,YY & r.. \\
\hline $1 \leqslant, 71$ & $\cdot, Y \leq$ & $r \cdot, \wedge 1$ & $\cdot, \cdot 1 \mathrm{~V}$ & IT,VY & $11, r v$ & r... \\
\hline$I V, \cdot T$ & $\theta, \cdot \leq 7$ & rr, 09 & $\cdot, \cdot T r$ & $r \cdot, \cdot r$ & IT,YY & Y.. T \\
\hline $1 \leqslant, \leqslant \Lambda$ & $\cdot, \cdot$ & $r 1, \cdot \Lambda$ & $1, \leqslant M$ & $r \wedge, \cdot r$ & $I V, \cdot V$ & Y...V \\
\hline $1 \leqslant, 01$ & $\cdot, 7$ & IT,TV & r, TOV & $M, r \varepsilon$ & 15,19 & $r \ldots 1$ \\
\hline $9, \mathrm{rA}$ &., $0 \leqslant$ & $1 \pi, \varepsilon 1$ & r,VOS & TY,rT & $9, Y$. & r...q \\
\hline$\varepsilon \cdot, r \mu$ & $\cdot, 0 \leq$ & 17,19 & 7,417 & $I V, O V$ & IY,VA & $r+1$. \\
\hline$r \wedge, Y r$ & $\cdot, \cdot+$ & 11,09 & $\varepsilon, \cdot T r$ & $r \cdot, \nabla \wedge$ & 11,10 & $r .11$ \\
\hline 17,91 & $\cdot, Y \cdot 1$ & $1 T, 11$ & $7,7 Y V$ & 19,90 & $1 \leqslant, 07$ & $r \cdot 1 r$ \\
\hline rr, rq & $\cdot, Y \cdot T$ & $1 T, \cdot 7$ & $r, \wedge \leqslant q$ & rT, & IT,VY & $r+1 r$ \\
\hline$T \leqslant, \leqslant \varepsilon$ & $\cdot, \leqslant r q$ & $17,9$. & r,90r & $\Gamma, Y V$ & $10,9 \leq$ & $r+1 \varepsilon$ \\
\hline$r 1,10$ & $\cdot, Y \mu T$ & $r \leq, 9 \leq$ & $7,19 \varepsilon$ & $\varepsilon 1,7 \varepsilon$ & $r \xi, \lambda r$ & $r .10$ \\
\hline$Y \cdot, \Sigma Y$ & $\cdot, 1 \vee 7$ & IV,qT & $0, \leqslant 01$ & Yo, $1 \leqslant$ & $1 \leqslant, r V$ & r.17 \\
\hline$Y . .70$ & $\because Y \leqslant Y$ & 11.77 & T. TV & $r \leqslant . \wedge 9$ & 10.7 & $r \cdot I V$ \\
\hline 19.05 & $\because \cdot \wedge \Lambda$ & IV.TY & T.11 & $Y \cdot . Y Y$ & $1 r_{.} \cdot \Lambda$ & المتوسط \\
\hline$\cdot, .01$ & $\cdot, \cdot 11$ & $\cdot, \mu_{-}$ & $\cdot, \leqslant \varepsilon$ & $. V \leq$ & $\cdot, 0$ & مقدار التغير السنوي \\
\hline$\cdot \Gamma \cdot$ & ${ }^{* *} Y \cdot, 0$ & ${ }^{*}, 17$ & ${ }^{* * *} r \cdot, 1 \wedge$ & *Y. . & $* *, 1 \Gamma$ & معدل النمو السنوي \% \\
\hline
\end{tabular}

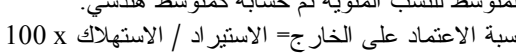




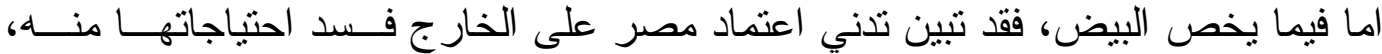

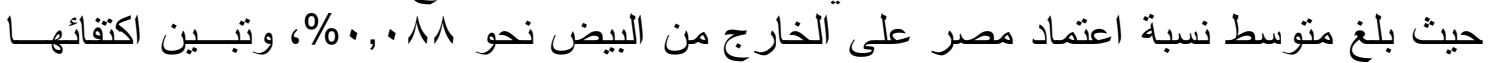

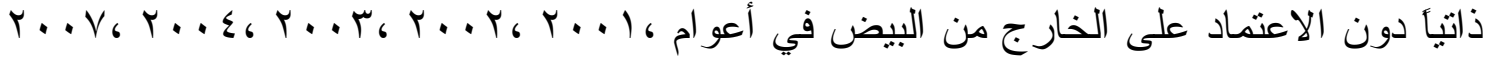

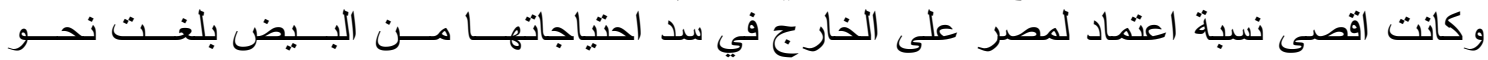

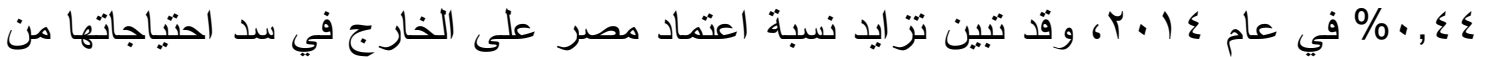

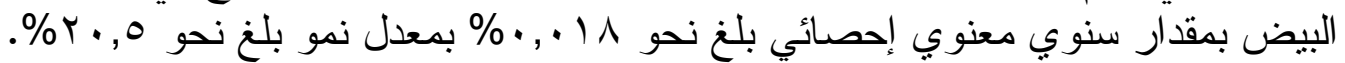

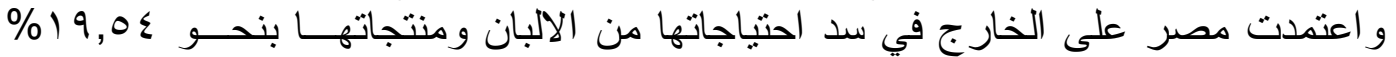

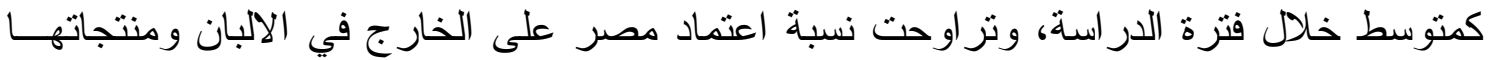

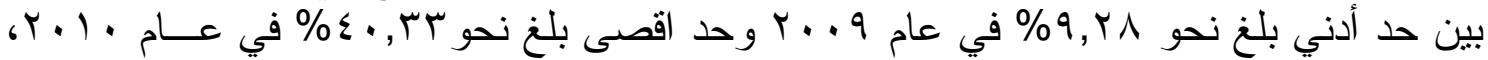

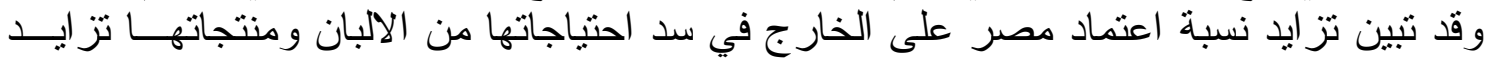
غير معنوي إحصائياً.

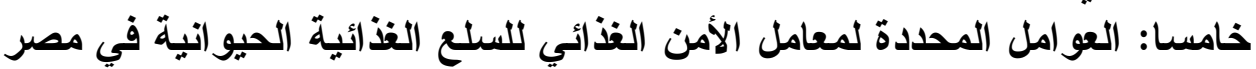

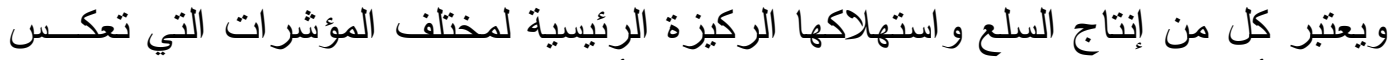

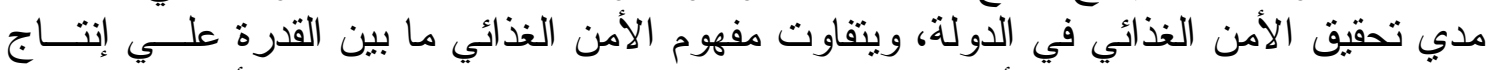

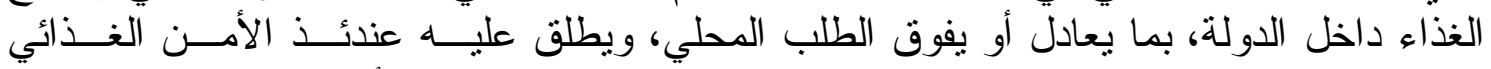

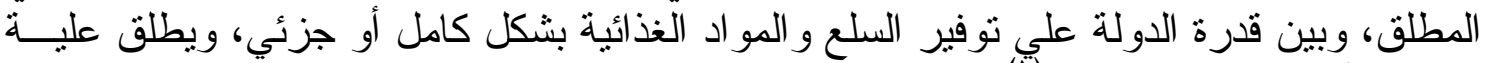

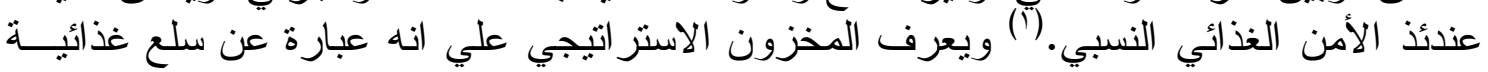

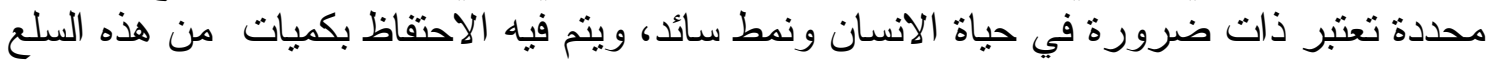

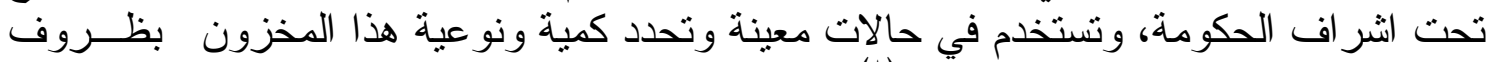
البلد نفسه وقر ار اته الفنية و الاقتصادية.

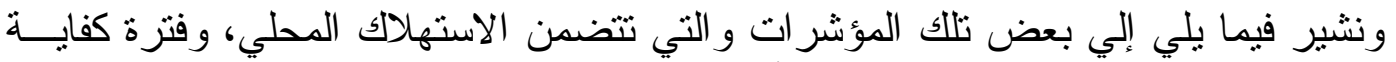

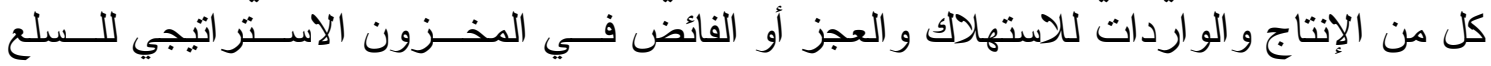

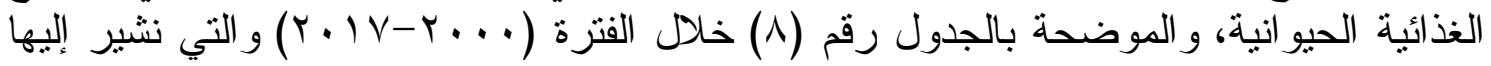

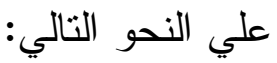

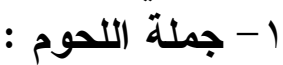

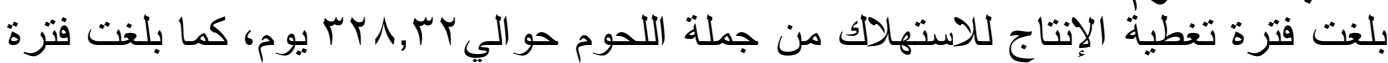

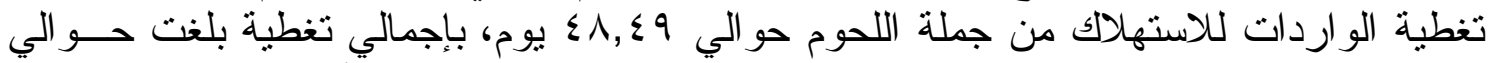
I,N,N

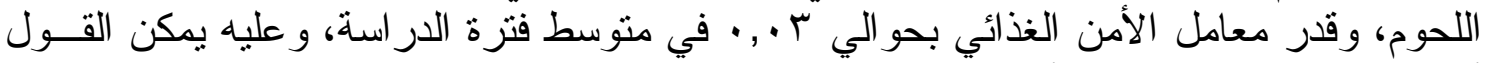
أن مصر نجحت في تحقيق أمنهم الغذائي النسبي معتمدة بشكل شبة كامل علي إني إنتاجها.

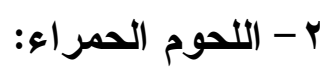

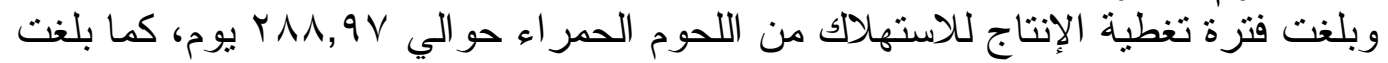

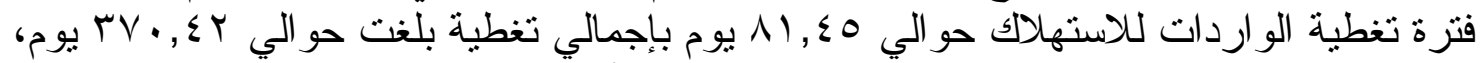

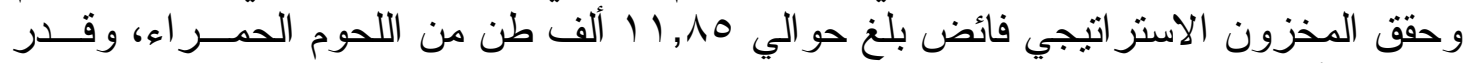

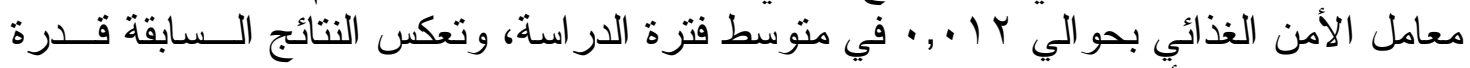
مصر علي تحقيق أمنها الغذائي النسبي من اللحوم الحمر اء معتمدة بشكل رئيسي علي الإئي الإنتاج.

$$
\text { ب - اللحوم البيضاء: }
$$

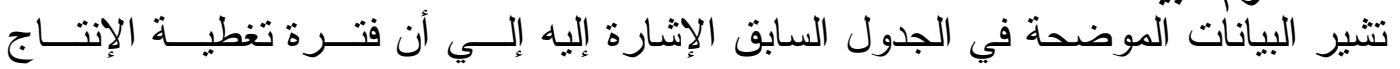

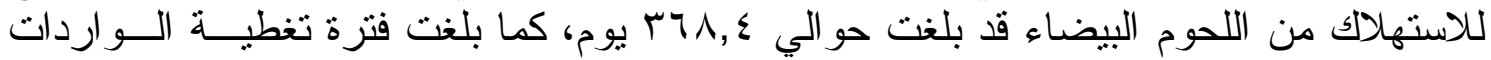




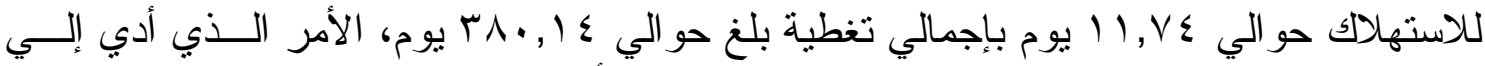

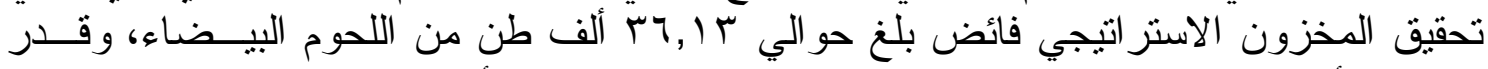

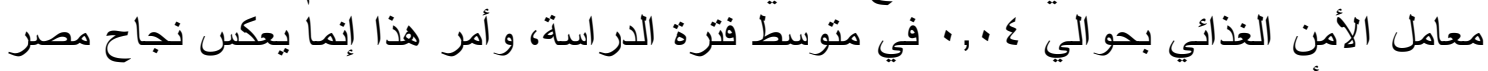
في تحقيق أمنها الغذائي النسبي بالاعتماد الكامل علي إنتاجهما من اللحوم البيضاء.

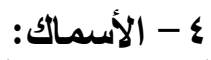

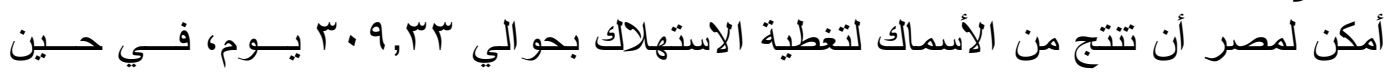

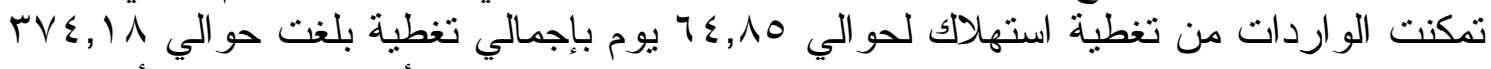

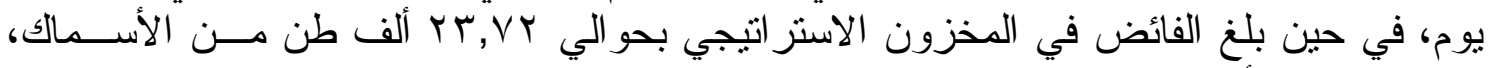

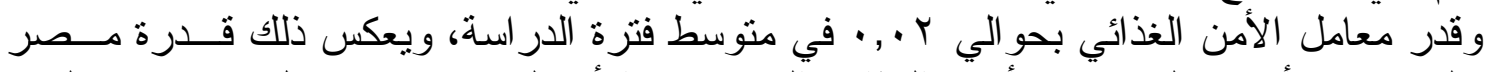

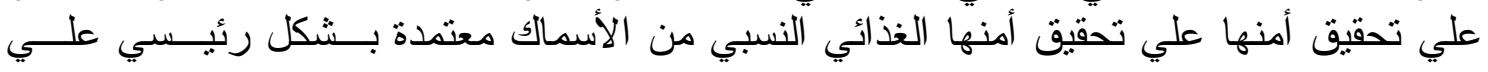

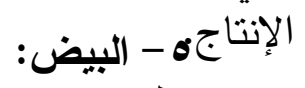

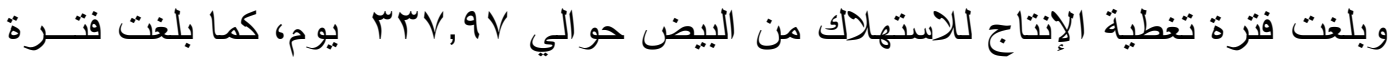

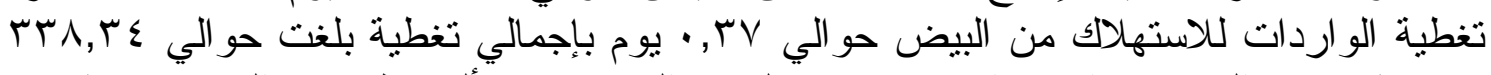

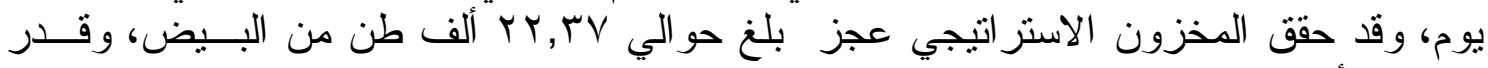

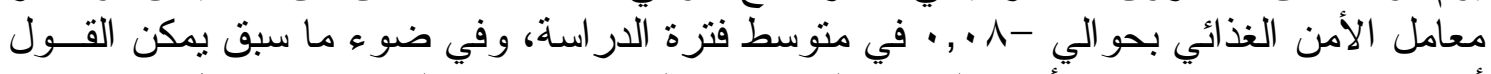

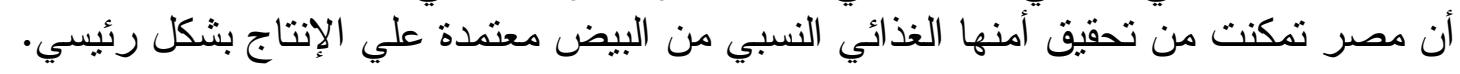

7 - الألبان ومنتجاتها:

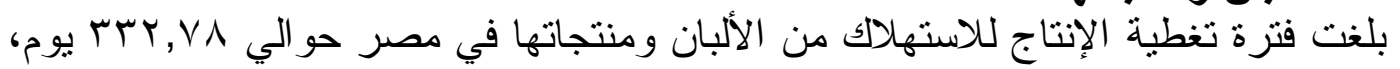

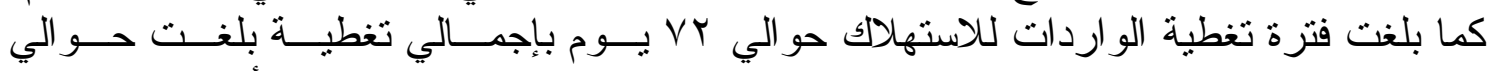

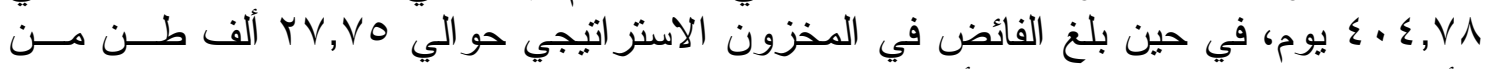

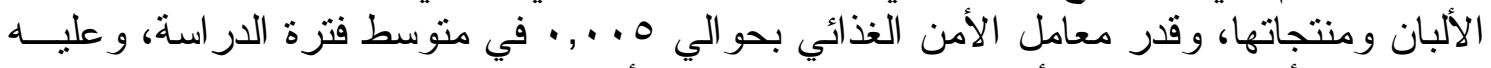

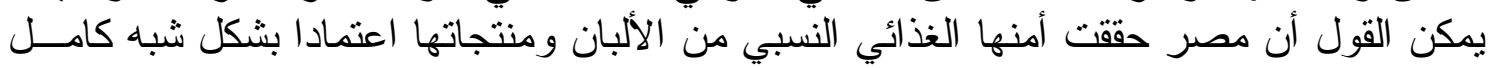
علي الإنتاج.

جدول ^ـ المخزون الاستراتيجي ومعامل الأمن الغذائي من أهم السلع والمجموعات السلعية الغذائية

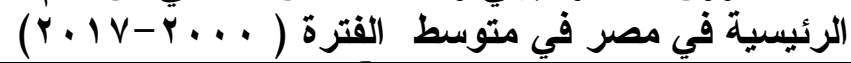

\begin{tabular}{|c|c|c|c|c|c|c|c|c|c|c|c|}
\hline \multirow{2}{*}{ 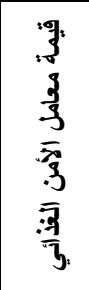 } & \multicolumn{2}{|c|}{ المخزون الاستراتيجي } & \multirow{2}{*}{ 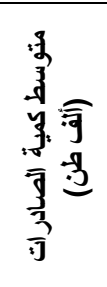 } & \multirow{2}{*}{ 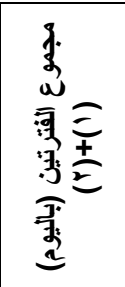 } & \multirow{2}{*}{ 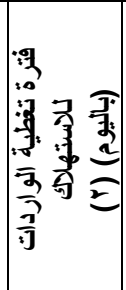 } & \multirow{2}{*}{ 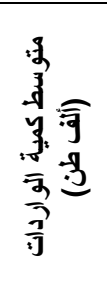 } & \multirow{2}{*}{ 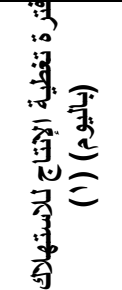 } & \multirow[b]{2}{*}{ 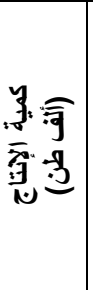 } & \multirow[b]{2}{*}{ 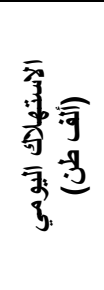 } & \multirow[b]{2}{*}{ 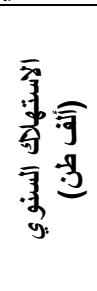 } & \multirow{2}{*}{ 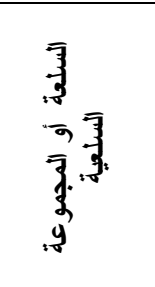 } \\
\hline & 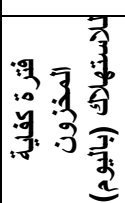 & 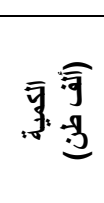 & & & & & & & & & \\
\hline$\cdot, \cdot r$ & $11, .1$ & 07,40 & $\varepsilon, 1 Y$ & $\checkmark Y, \wedge 1$ & $\varepsilon \wedge, \varepsilon 9$ & $r \leqslant \Lambda, Y_{O}$ & TYA,TY & 1711 & $0,1 Y$ & $1 \wedge V \varepsilon$ & جملة اللحو. \\
\hline & $\{, 01$ & 11,10 & $r, \varepsilon$. & $r V_{\cdot}, \varepsilon r$ & $11, \leqslant 0$ & $r \mid \varepsilon, r r$ & 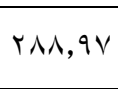 & VI. & אז, & 971 & الحمر اءع \\
\hline$\cdot, \cdot \Sigma$ & $1 \leqslant, \leqslant 0$ & 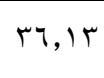 & $1, V Y$ & $\left\lceil\Lambda_{0},{ }_{1}, \varepsilon\right.$ & $11, V \varepsilon$ & rq,ro & $r \uparrow \Lambda, \varepsilon$ & 941 & $r, 0$ & $91 \%$ & البضضاء \\
\hline$\cdot, \cdot Y$ & $4, \pi$ &,$V Y$ & $1 \cdot, v 1$ & $\varepsilon, 1 \wedge$ & $T \varepsilon, \wedge 0$ & YY & $r$ & 117. & $r, v_{0}$ & ITVI & الاسماك \\
\hline,$\cdots A_{-}$ & 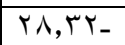 & $Y Y, r V_{-}$ & $1, r Y$ & $r r \Lambda, r \varepsilon$ & $\cdot, r V$ & $\cdot, r q$ & TrV,qV & YTV & $\cdot$, , & r^q & البيض \\
\hline & $1, V V$ & $Y V, V O$ & 097 & $\varepsilon \cdot \varepsilon, \vee \wedge$ & VY & 1119 & ג & oris & 10,71 & OVTV & والالبان \\
\hline
\end{tabular}

ا - - جامعة الدول العربية، المنظمة العربية للتنمية الزر اعية، الكتاب السنوي للإحصاءات الزر اعية العربية، الخرطوم، أعداد مختلفة.

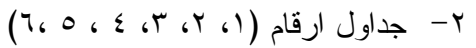




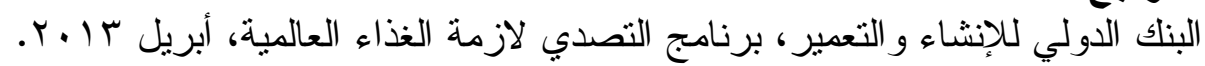

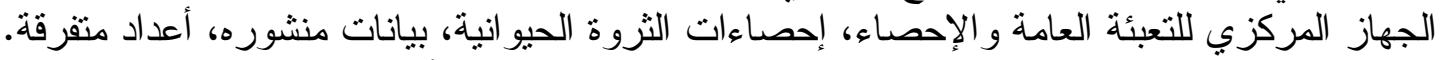
الجهاز المركزي للتعبئة العامة و الإحصاء، الكتابة الإحاءة الإحصائي السنوي، العاءة أعداد مختلفة. الجهاز المركزي للتعبئة العامة و الإحصاء، نشرة الإعة التجارة الخارجية، الإلة العداد مختلفة.

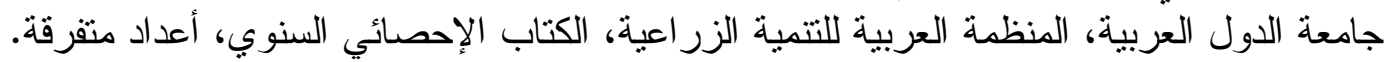

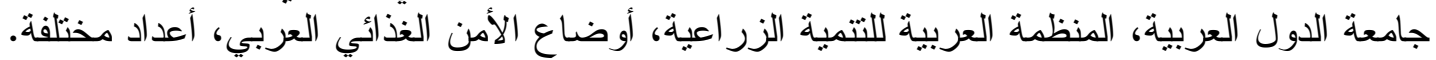

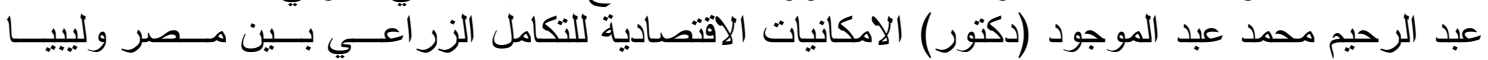

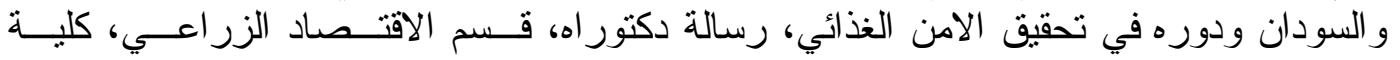

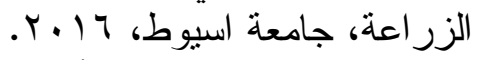

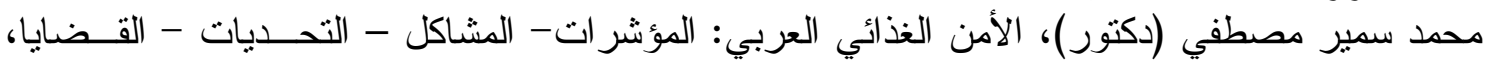

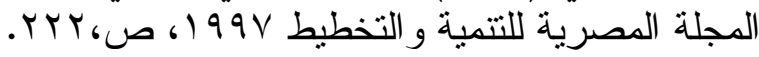

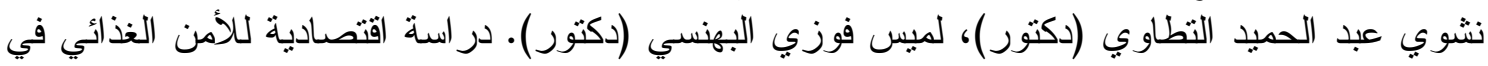

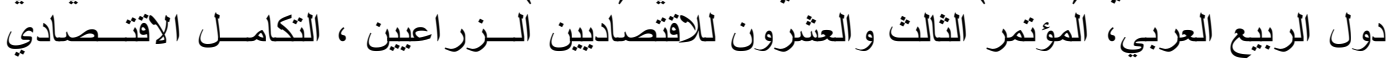

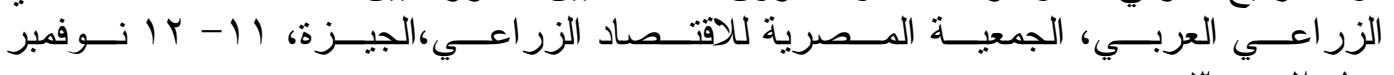
. وزارة الزر اعة و استصلاح الأر اضي، قطاع الثئون الاقتصادية، الإدارة المركزية للاقتصاد الزر اعـي، نشرة الميزان الغذائي، أعداد مختلفة. الأحس،

UN Standing Committee on Nutrition, 2004. Annual Report. 


\title{
An Analytical Study of the Food Security Situation of Animal Food
} Commodities in Egypt

\author{
Abd El-Rahim M. Abd El- Mowgod ${ }^{1}$ and Hassan M. Radwan ${ }^{2}$ \\ ${ }^{1}$ Agric. Econ. Dept., Fac. of Agric., Assiut Univ., Assiut \\ ${ }^{2}$ Higher Institute for Cooperation and Agricultural Extension in Assiut
}

\section{Summary}

The food issue is one of the most important strategic issues of concern to Egypt, which strives to increase the rates of self-sufficiency, reduce the food gap and achieve food security from agricultural and animal commodities. And the research aims to study and analyze the productive and consumption capacities of livestock, poultry and fish and their repercussions in an attempt to achieve food security in Egypt. The research results showed that the percentage of selfsufficiency in total meat decreased annually by about $0.75 \%$, from red meat annually by about $1.11 \%$, and from white meat by about $0.42 \%$. While the percentage of self-sufficiency in Milk \& Dairy Production increased annually by about $0.63 \%$. Egypt relied on abroad to meet its needs of total meat by about $12,08 \%$, about $20.22 \%$ of red meat, about $2.18 \%$ of white meat, about $17.62 \%$ of fish, about $0.08 \%$ of eggs, and about $19.54 \%$ of Milk and dairy Production.

The data indicate a decrease in the food security coefficient of animal food commodities in Egypt. The average food security coefficient for the total meat group was about 0.03 , about 0.012 in red meat, and in white meat by about 0.04 , while it reached about 0.02 in fish, as it reached about - 0.08 in eggs, while it reached about 0.005 in Milk and dairy products. The research recommends raising the strategic stock of animal food commodities in Egypt, by relying on increasing local production by paying attention to the animal production sector and providing and encouraging investment in it. 